TRANSACTIONS OF THE

AMERICAN MATHEMATICAL SOCIETY

Volume 352, Number 4, Pages 1493-1525

S 0002-9947(99)02463-0

Article electronically published on November 18, 1999

\title{
RATIONAL HOMOTOPY THEORY FOR NON-SIMPLY CONNECTED SPACES
}

\author{
ANTONIO GÓMEZ-TATO, STEPHEN HALPERIN, AND DANIEL TANRÉ
}

\begin{abstract}
We construct an algebraic rational homotopy theory for all connected CW spaces (with arbitrary fundamental group) whose universal cover is rationally of finite type. This construction extends the classical theory in the simply connected case and has two basic properties: (1) it induces a natural equivalence of the corresponding homotopy category to the homotopy category of spaces whose universal cover is rational and of finite type and (2) in the algebraic category, homotopy equivalences are isomorphisms. This algebraisation introduces a new homotopy invariant: a rational vector bundle with a distinguished class of linear connections.
\end{abstract}

The rationalisation of an abelian group $\Gamma$ is the rational vector space $\Gamma \otimes \mathbb{Q}$, together with the obvious homomorphism $\Gamma \rightarrow \Gamma \otimes \mathbb{Q}$. Rational homotopy theory begins with the introduction by Sullivan [20] of a geometric analogue: with any simply connected CW space $X$ there is associated a continuous map $f: X \rightarrow X_{\mathbb{Q}}$ with $X_{\mathbb{Q}}$ simply connected, and such that $\pi_{i}\left(X_{\mathbb{Q}}\right)=\pi_{i}(X) \otimes \mathbb{Q}$ and $\pi_{i}(f)$ is the rationalisation. The homotopy type of $X_{\mathbb{Q}}$ is called the rational homotopy type of $X$, the space $X_{\mathbb{Q}}$ is called the rationalisation of $X$, and $X$ is called a rational space if the groups $\pi_{i}(X)$ themselves are rational - in which case $f$ is a homotopy equivalence.

A principal feature of rational homotopy theory in the simply connected case, as developed by Quillen [18] and Sullivan [21, is that the geometric construction $X_{\mathbb{Q}}$ can be replaced by an equivalent algebraic construction. Sullivan's approach consists of three steps. First, a simplicial commutative cochain algebra $\left\{A_{P L}(n), \partial_{i}, s_{i}\right\}$ is used to define a functor $A_{P L}(-)$ from connected simplicial sets (and topological spaces) to the category $\mathcal{A}$ of commutative cochain algebras $A$ satisfying $H^{0}(A)=\mathbb{Q}$. Second, a distinguished class of these cochain algebras is introduced, now called minimal Sullivan algebras. Any cochain algebra $A \in \mathcal{A}$ admits a quasi-isomorphism $M \stackrel{\simeq}{\rightarrow} A$ from a uniquely determined minimal Sullivan algebra, the minimal Sullivan model of $A$. If $X$ is a connected topological space, then the minimal model $M_{X}$ of $A_{P L}(X)$ is a Sullivan model for $X$. Finally within $\mathcal{A}$ the full subcategory $\mathcal{M}$ of minimal Sullivan algebras is equipped with a notion of homotopy. A homotopy preserving functor \langle\rangle is then constructed from $\mathcal{M}$ to

Received by the editors November 20, 1997.

1991 Mathematics Subject Classification. Primary 55P62, 55R25.

Key words and phrases. Fundamental group, simplicial set, Sullivan model, rational homotopy, rational vector bundle, linear connections.

The first author's research was partially supported by an "Action Intégrée" and a Xunta of Galicia grant, the second author's research was partially supported by a NATO grant and a NSERC grant and the third author's research was partially supported by a NATO grant. 
simplicial sets and, if $X$ is simply connected with rational homology of finite type, then $\left\langle M_{X}\right\rangle$ is a rationalisation of $X$.

An important feature is that homotopy equivalences in $\mathcal{M}$ are automatically isomorphisms. Thus the model $M_{X}$ for $X$ has the two fundamental properties [21]:

(1) The passage $X \leadsto M_{X}$ does not lose any rational homotopy theoretic information.

(2) The isomorphism class of $M_{X}$ (and any of its invariants) is a rational homotopy invariant of $X$.

Subsequent applications of this machine have led to the discovery of a wide variety of geometric phenomena for simply connected spaces, sometimes of a purely rational nature, but often suggesting new theorems about their integral homotopy invariants.

For non-simply connected CW spaces, the appropriate geometric rationalisation is supplied by the work of Bousfield and Kan [2]: for any such space $X$ there is a natural fibration $\tilde{X} \rightarrow X \rightarrow B \pi_{1}(X)$ whose fibre is homotopy equivalent to the universal cover of $X$. Bousfield and Kan provide a fibrewise rationalisation $\tilde{X}_{\mathbb{Q}} \rightarrow X_{\mathbb{Q}} \stackrel{\rho}{\rightarrow} B \pi_{1}(X)$ whose fibre is the classical rationalisation of the simply connected space $\tilde{X}$. There is now a general consensus that this is the correct notion of rationalisation, so that $X$ is rational if and only if its higher homotopy groups, $\pi_{i}(X), i \geq 2$, are rational vector spaces. Thus the rationalisation of $X$ consists of three pieces of data:

- The classical rationalisation, $\tilde{X}_{\mathbb{Q}}$, of the universal cover.

- The fundamental group, $\pi_{1}(X)$.

- The twisting of $\tilde{X}_{\mathbb{Q}}$ over $B \pi_{1}(X)$ into the fibration $X_{\mathbb{Q}}$.

(Note by contrast that Sullivan's extension of the classical theory to nilpotent spaces has the effect of rationalising the fundamental group. In this case, it is easy to see that $X_{\mathbb{Q}}$ is the pull-back of his rationalisation, $X_{S \mathbb{Q}}$, over the induced map $\left.B \pi_{1}(X) \rightarrow B\left(\pi_{1}(X)_{\mathbb{Q}}\right).\right)$

Thus one approach to an algebraic description of $X_{\mathbb{Q}}$ is to model the twist of the Sullivan model of $\tilde{X}$ over $B \pi_{1}(X)$. (Representations of $\pi_{1}(X)$ in the model do not always suffice as we see in Example 6.7]) This problem is solved first in 15 in the smooth category, and then by Brown and Szczarba in 4 as follows. The fibration $X \rightarrow B \pi_{1}(X)$ pulls back to a fibration $\hat{X} \rightarrow E \pi_{1}(X)$ of $\pi_{1}(X)$ spaces, where $E \pi_{1}(X)$ is the universal cover of $B \pi_{1}(X)$; in particular, $\hat{X} \simeq \tilde{X}$. The fibration $\hat{X} \rightarrow E \pi_{1}(X)$ admits a $\pi_{1}(X)$-equivariant Postnikov decomposition, which is used in 4 to construct a $\pi_{1}(X)$-equivariant Sullivan model. Then in [4] Sullivan's geometric realisation functor is applied to this model to construct $X_{\mathbb{Q}}$, thereby establishing that the model determines the rational homotopy type of $X$. Moreover, the work in [4] is carried out in the context of the category $\mathcal{F}_{\mathbf{K}}^{f}$ of general Kan fibrations

$$
\xi: \mathbf{F} \rightarrow \mathbf{X} \rightarrow \mathbf{K}
$$

with $\mathbf{F}$ simply connected and each $\pi_{i}(\mathbf{F}) \otimes \mathbb{Q}$ finite dimensional, and with $\mathbf{K}$ any connected simplicial set.

In this paper we extend the work in [4] to a complete analogue of Sullivan's approach in the simply connected case, working as in 4 in the context of the category $\mathcal{F}_{\mathbf{K}}^{f}$. First, we introduce a new algebraic category $\mathcal{A}_{\mathbf{K}}$ of local systems of commutative cochain algebras over $\mathbf{K}$ (it plays the role of $\mathcal{A}$ in the classical case) and 
we use $A_{P L}(*)$ to construct a functor from $\mathcal{F}_{\mathbf{K}}^{f}$ to $\mathcal{A}_{\mathbf{K}}$. Within $\mathcal{A}_{\mathbf{K}}$ we distinguish a subcategory $\mathcal{M}_{\mathbf{K}}$ of minimal algebras and we show that any element of $\mathcal{A}_{\mathbf{K}}$ has a minimal Sullivan model, whose geometric realisation is just the rationalisation $\mathbf{X}_{\mathbb{Q}} \rightarrow \mathbf{K}$. Finally, we show that geometric realisation is an equivalence of homotopy categories from $\mathcal{M}_{\mathbf{K}}$ to $\mathcal{F}_{\mathbf{K}}^{f}$.

It is easy to see that the pull-back of our minimal model to the universal cover $\tilde{\mathbf{K}}$ is a minimal model as constructed in [4], and that the geometric realisation here coincides with the realisation in 4 for the pull-back.

The essential part of the paper, however, is the analysis in $\S 1-\S 3$ of the structure of minimal models in this setting. This requires that we introduce a new notion: 'rational vector bundles' over a simplicial set. These (the $R$-modules of $\S 1$ ) are the analogue of classical vector bundles and indeed if rational is replaced by real and the simplicial set is finite, then the two notions coincide. Then a minimal model for the fibration $(*)$ consists of:

- a rational vector bundle $\left(\Lambda Y, D_{0}\right)$ over $\mathbf{K}$ whose fibre at the base point is the classical minimal model of $\mathbf{F}$.

- a 'homotopy gauge class' of distinguished linear connections $D_{1}$ in the vector bundle $\left(\Lambda Y, D_{0}\right)$.

- a differential $D=D_{0}+D_{1}+D_{2} \cdots$ in the differential forms on $\mathbf{K}$ with values in $\Lambda Y$, such that $D_{i}$ raises the form degree by $i$.

With the aid of this analysis we show in particular that a minimal Sullivan model is unique up to isomorphism. This completes the analogy with the simply connected case.

It also exhibits the vector bundle $\left(\Lambda Y, D_{0}\right)$ and the homotopy gauge class of connections $D_{1}$ as new homotopy invariants of the fibration. In particular, the geometry of the connection $D_{1}$ is an important part of the description of the twisting of $\mathbf{F}_{\mathbb{Q}}$ over $\mathbf{K}$. For instance, we construct in Example 6.7 a fibration in which $\pi_{1}(\mathbf{K})$ cannot act on the minimal model of $\mathbf{F}_{\mathbb{Q}}$ and induce the classical action in $H^{*}\left(\mathbf{F}_{\mathbb{Q}}\right)$. This implies (as we show in a subsequent paper) that no distinguished linear connection $D_{1}$ can be flat although in this example the vector bundle does admit other flat connections.

In [21] Sullivan pointed out that $C^{\infty}$-differential forms could be used instead of rational differential forms to provide a 'real' analogue of rational homotopy theory. This also carries over completely to the non-simply connected case, where we may replace $A_{P L}(*)$ by the simplicial cochain algebra $A_{D R}(*)$ that assigns to each $n$ the algebra of $C^{\infty}$ differential forms on $\Delta^{n}$. Here we find real vector bundles and linear connections over a simplicial set. The case that $\mathbf{K}$ is itself a smooth manifold was already dealt with in [15, where the vector bundles and linear connections are the classical objects.

In fact, we work with an arbitrary simplicial commutative cochain algebra $A(*)$ over any field $\mathbf{k}$ of characteristic zero, provided that $A(*)$ satisfies the four basic classical properties: $\left(A(\mathbf{p t})=\mathbf{k}, A(n)=A^{0}(n) \otimes \Lambda\left(d t_{1}, \ldots, d t_{n}\right)\right.$, for elements $t_{i} \in A^{0}(n), A(*)$ is extendable (Definition 1.4), $H(A(n))=\mathbf{k}$ and one further property: if $f \in A^{0}(n)$, then $d f=f \omega$ implies $f=0$ or $f$ is invertible. Both $A_{P L}(*)$ and $A_{D R}(*)$ then arise as particular examples.

Our program is carried out in Sections 1-7 below, whose headings are selfexplanatory:

$\S 1$. Linear algebra of $R$-modules 
§2. $R$ minimal models

$\S 3$. A minimal models

$\S 4$. Homotopy

$\S 5$. Kan fibrations

$\S 6$. Non-simply connected rational homotopy theory

$\S 7$. Applications, examples and problems.

\section{Linear Algebra of $R$-Modules}

Let $\mathbf{K}$ be a connected simplicial set. The $n$-skeleton of $\mathbf{K}$ is the simplicial set $\mathbf{K}^{(n)}$ generated by the non-degenerate simplices of dimension $\leq n$, and $\mathbf{K}$ is $(n+1)$ reduced if $\mathbf{K}$ has a single vertex and no other non-degenerate simplices of dimension $\leq n$. We say that $\mathbf{K}$ is finite if it has only finitely many non-degenerate simplices; i.e. if its geometric realisation $|\mathbf{K}|$ is a finite polyhedron. We denote by $\boldsymbol{\Delta}^{n}$ the standard simplicial $n$-simplex, by $\partial \boldsymbol{\Delta}^{n}$ its $(n-1)$-skeleton and by $\boldsymbol{\Delta}^{n, k}$ the subsimplicial set of $\partial \boldsymbol{\Delta}^{n}$ obtained by removing the $k$-th $(n-1)$-face. The dimension $n$ of a simplex $\sigma$ of $\mathbf{K}$ is written $|\sigma|=n$, and we identify $\sigma$ with the induced simplicial map: $\sigma: \boldsymbol{\Delta}^{n} \rightarrow \mathbf{K}$.

We also recall [16] that a Kan fibration is a simplicial map $\mathbf{K} \rightarrow \mathbf{K}^{\prime}$ such that for each commutative diagram of simplicial maps

$$
\begin{array}{cccc}
\boldsymbol{\Delta}^{n, k} & \stackrel{f}{\rightarrow} & \mathbf{K} & \\
\downarrow & & \downarrow & \\
\boldsymbol{\Delta}^{n} & \stackrel{g}{\rightarrow} & \mathbf{K}^{\prime} & (0 \leq k \leq n),
\end{array}
$$

there exists a map $\boldsymbol{\Delta}^{n} \rightarrow \mathbf{K}$ extending $f$ and lifting $g$. When $\mathbf{K}^{\prime}=\boldsymbol{\Delta}^{0}, \mathbf{K}$ is called a Kan complex.

Vector bundles (real or complex) over $|\mathbf{K}|$ are a classical concept. Our goal in this section is to describe linear objects over $\mathbf{K}$ with projectivity properties analogous to those of the module of cross-sections of vector bundles over compact spaces, the role of continuous functions being played by a local system $R$ of rings on $\mathbf{K}$ (for example, the polynomial functions of Sullivan [21, $\S 7]$ ). A basic notion will be that of local systems over $\mathbf{K}$, as described in [13, Chapter 12]:

Definition 1.1. Let $\mathcal{C}$ be a category of sets, with pullbacks, possibly graded or with additional structure.

(1) A local system $F$ on $\mathbf{K}$, with values in $\mathcal{C}$, is:

(i) a family of objects $F_{\sigma} \in \mathcal{C}$, indexed by the simplices $\sigma$ of $\mathbf{K}$; together

with

(ii) a family of morphisms $\partial_{i}: F_{\sigma} \longrightarrow F_{\partial_{i} \sigma}, s_{i}: F_{\sigma} \longrightarrow F_{s_{i} \sigma}$, called the face and degeneracy operators, and satisfying the usual commutation relations.

(2) A morphism $\psi: F \longrightarrow G$ between local systems is a family of morphisms $\psi_{\sigma}: F_{\sigma} \longrightarrow G_{\sigma}$, indexed by the simplices $\sigma$ of $\mathbf{K}$, and compatible with the face and degeneracy operators.

(3) The global section functor, $\Gamma$, associates to a local system $F$ on $\mathbf{K}$ the object of $\mathcal{C}$ whose elements are the functions that assign to each simplex $\sigma \in \mathbf{K}$ an element $\Phi_{\sigma} \in F_{\sigma}$, and which are compatible with the face and degeneracy operators. For any simplex $\sigma$ the evaluation morphism $\Gamma(F) \rightarrow F_{\sigma}$ in $\mathcal{C}$ is defined by $\Phi \mapsto \Phi_{\sigma}$.

When $\mathcal{C}=\left\{\mathcal{C}^{p}\right\}$ is a category of graded sets, then $F=\left\{F^{p}\right\}$ and $\Gamma(F)$ is the graded object defined by $\Gamma(F)^{p}=\Gamma\left(F^{p}\right)$. 
(4) The pull-back of $F$ via a simplicial map $\mathbf{u}: \mathbf{L} \rightarrow \mathbf{K}$ is the local system $F^{\mathbf{u}}$ on $\mathbf{L}$ defined by $\left(F^{\mathbf{u}}\right)_{\sigma}=F_{\mathbf{u}(\sigma)}$. When $\mathbf{L} \subset \mathbf{K}$, we write instead $F_{\mid \mathbf{L}}$ and call it the restriction of $F$ to $\mathbf{L}$. There is an obvious pull-back or restriction morphism $\Gamma(F) \rightarrow \Gamma\left(F^{\mathbf{u}}\right)$. When $\mathbf{L} \subset \mathbf{K}$ and $\mathcal{C}$ is an abelian category, the kernel of the restriction morphism is the object $\Gamma(F ; \mathbf{K}, \mathbf{L})=\left\{\Phi \in \Gamma(F) \mid \Phi_{\sigma}=0, \sigma \in \mathbf{L}\right\}$.

In particular, if $\sigma \in \mathbf{K}_{n}$, we denote by $F^{\sigma}$ the pull-back of $F$ via the simplicial map $\sigma: \boldsymbol{\Delta}^{n} \rightarrow \mathbf{K}$. We identify $\Gamma\left(F^{\sigma}\right)=F_{\sigma}$ via the $\mathcal{C}$-isomorphism $\Phi \mapsto \Phi_{\boldsymbol{\Delta}_{n}}$. By analogy, we adopt the notation $\Gamma\left(F_{\mid \partial \boldsymbol{\Delta}^{n}}^{\sigma}\right)=F_{\partial \sigma}$ and (when $\mathcal{C}$ is an abelian category) $\Gamma\left(F^{\sigma} ; \boldsymbol{\Delta}^{n}, \partial \boldsymbol{\Delta}^{n}\right)=F_{\sigma, \partial \sigma}$.

Definition 1.2. (1) If $F$ is a local system of differential $\mathbb{Z}$-modules the homology of $F$ is the local system $H(F)=\left\{H\left(F_{\sigma}\right), H\left(\partial_{i}\right), H\left(s_{j}\right)\right\}$.

(2) A morphism $\varphi: C \rightarrow C^{\prime}$ between two such local systems is called a quasiisomorphism and written $C \stackrel{\cong}{\rightrightarrows} C^{\prime}$ if $H(\varphi)$ is an isomorphism. It is called a $\Gamma$-quasiisomorphism if $H(\Gamma(\varphi))$ is an isomorphism.

Remark 1.3 ([13 Lemma 12.17]). Given $\Phi \in \Gamma\left(F_{\mid \mathbf{K}^{(n)}}\right)$ together with elements $v_{\sigma} \in F_{\sigma}$ for each non-degenerate $(n+1)$ simplex $\sigma$ and satisfying $\partial_{i} v_{\sigma}=\Phi_{\partial_{i} \sigma}$, then there is a unique $\Psi \in \Gamma\left(F_{\mid \mathbf{K}^{(n+1)}}\right)$ extending $\Phi$ and satisfying $\Psi_{\sigma}=v_{\sigma}$.

Definition 1.4. A local system $F$ on $\mathbf{K}$ is:

(1) extendable if, for each simplex $\sigma \in \mathbf{K}$, the restriction morphism $F_{\sigma} \rightarrow F_{\partial \sigma}$ is surjective;

(2) locally constant if the face and degeneracy operators are isomorphisms;

(3) constant if for some $G \in \mathcal{C}, F_{\sigma}=G$ for each $\sigma \in \mathbf{K}$, and each $\partial_{i}$ and $s_{i}$ is the identity map of $G$.

We recall from [13, 12.21] that if $F$ is extendable, then for any $\mathbf{L} \subset \mathbf{K}, \Gamma(F) \rightarrow$ $\Gamma\left(F_{\mid \mathbf{L}}\right)$ is surjective. When $\mathcal{C}$ is abelian, we then have the short exact sequence:

$$
0 \longrightarrow \Gamma(F ; \mathbf{K}, \mathbf{L}) \longrightarrow \Gamma(F) \longrightarrow \Gamma\left(F_{\mid \mathbf{L}}\right) \longrightarrow 0 .
$$

Now let $R$ be a local system of rings over $\mathbf{K}$, so that $\Gamma(R)$ is a ring.

Definition 1.5. An $R$-module, $V$, is a local system of abelian groups over $\mathbf{K}$ such that each $V_{\sigma}$ is an $R_{\sigma}$-module, and scalar multiplication $R_{\sigma} \otimes_{\mathbb{Z}} V_{\sigma} \rightarrow V_{\sigma}$ is compatible with the face and degeneracy operators. A morphism of $R$-modules is called an $R$-linear map.

In the same way we define graded $R$-modules, differential graded $R$-modules and, when $R$ is commutative, differential graded $R$-algebras, ...; in each case the extra structure being required to commute with scalar multiplication from $R$.

Remark 1.6. (i) $R$-modules are local systems of $\Gamma(R)$-modules, via the evaluation homomorphisms $\Gamma(R) \rightarrow R_{\sigma}$.

(ii) The category of $R$-modules is an abelian category with products and tensor products: $(V \oplus W)_{\sigma}=V_{\sigma} \oplus W_{\sigma} ;\left(\Pi_{i} V(i)\right)_{\sigma}=\Pi_{i} V(i)_{\sigma} ;\left(V \otimes_{R} W\right)_{\sigma}=V_{\sigma} \otimes_{R_{\sigma}} W_{\sigma}$. A sequence $U \stackrel{\phi}{\rightarrow} V \stackrel{\psi}{\rightarrow} W$ of $R$-modules is exact if and only if each $U_{\sigma} \stackrel{\phi_{\sigma}}{\rightarrow} V_{\sigma} \stackrel{\psi_{\sigma}}{\rightarrow} W_{\sigma}$ is exact.

(iii) If $V$ is an $R$-module, then $\Gamma(V)$ is a $\Gamma(R)$-module via $(f . \Phi)_{\sigma}=f_{\sigma} . \Phi_{\sigma}, f \in$ $\Gamma(R), \Phi \in \Gamma(V)$. A left adjoint to this functor is defined as follows: Given a $\Gamma(R)$-module $M$, we define the $R$-module $R \otimes_{\Gamma} M$ by $\left(R \otimes_{\Gamma} M\right)_{\sigma}=R_{\sigma} \otimes_{\Gamma(R)} M$, where $\Gamma(R)$ acts on $R_{\sigma}$ via the evaluation morphism. When $\mathbf{K}=\boldsymbol{\Delta}^{n}$, we have 
identified $\Gamma(R)=R_{\Delta^{n}}$ via evaluation. Thus, for each $R_{\Delta^{n}}$-module $V$ we obtain the $R$-module $R \otimes_{\Gamma} V$ on $\boldsymbol{\Delta}^{n}$.

(iv) $R$ itself is an $R$-module.

(v) If $\mathbf{u}: \mathbf{L} \rightarrow \mathbf{K}$ is a simplicial map and $V$ is an $R$-module, then the pull-back $V^{\mathbf{u}}$ is an $R^{\mathbf{u}}$-module.

(vi) If $V$ and $W$ are $R$-modules, we denote by $R$ - $\operatorname{lin}(V, W)$ the $\mathbb{Z}$-module of $R$-linear maps from $V$ to $W$. Note that a natural isomorphism $R$ - $\operatorname{lin}(R, V) \stackrel{\cong}{\rightrightarrows}$ $\Gamma(V)$ is given by $f \mapsto\left\{f_{\sigma}\left(1_{\sigma}\right)\right\}$ and that, more generally, $R$ - $\operatorname{lin}\left(\bigoplus_{i} R e_{i}, V\right) \stackrel{\cong}{\rightrightarrows}$ $\Pi_{i}\left(e_{i} \times \Gamma(V)\right)$.

Lemma 1.7. Let $(V(\alpha))_{\alpha \in J}$ be a family of $R$-modules. Then the canonical map $\gamma: \bigoplus_{\alpha} \Gamma(V(\alpha)) \longrightarrow \Gamma\left(\bigoplus_{\alpha} V(\alpha)\right)$ is injective. If $J$ or $\mathbf{K}$ is finite, then $\gamma$ is an isomorphism.

Lemma 1.8. The direct sum of $R$-modules, $V(\alpha)$, is extendable if and only if each $V(\alpha)$ is extendable.

Proof. Since extendability of a local system $F$ is defined in terms of the pullbacks $F^{\sigma}$ on $\boldsymbol{\Delta}^{|\sigma|}$ and $\boldsymbol{\Delta}^{|\sigma|}$ is finite, the lemma follows at once from Lemma 1.7 .

Definition 1.9. An $R$-module $V$ is:

(1) free if it is a direct sum of copies of $R$.

(2) locally free if, for each $\sigma \in \mathbf{K}, V^{\sigma}$ is a free $R^{\sigma}$-module.

Lemma 1.10. Suppose $Y$ is a locally free $R$-module and let $M$ be any $R$-module. Then

(i) a local system of abelian groups, $\operatorname{Hom}_{R}(Y, M)$, is uniquely determined by the conditions: $\left[\operatorname{Hom}_{R}(Y, M)\right]_{\sigma}=\operatorname{Hom}_{R_{\sigma}}\left(Y_{\sigma}, M_{\sigma}\right) ; \partial_{i}\left(f_{\sigma}\right) \circ \partial_{i}=\partial_{i} \circ f_{\sigma} ; s_{j}\left(f_{\sigma}\right) \circ s_{j}=$ $s_{j} \circ f_{\sigma}$. If $M$ is extendable, so is $\operatorname{Hom}_{R}(Y, M)$.

(ii) $R-\operatorname{lin}(Y, M)=\Gamma\left(\operatorname{Hom}_{R}(Y, M)\right)$.

Proof. (i) For any $\sigma \in \mathbf{K}$ we may write $Y^{\sigma}=R^{\sigma} \otimes_{\Gamma} X(\sigma)$, where $X(\sigma)$ is a free $R_{\sigma^{-}}$ module on a basis $\left\{x_{\alpha}\right\}$. Hence the $Y_{\partial_{i} \sigma}$ (resp. $Y_{s_{j} \sigma}$ ) are generated as $R_{\partial_{i} \sigma}$ (resp. $R_{s_{j} \sigma}$ ) modules by $\partial_{i}\left(Y_{\sigma}\right)$ (resp. $s_{j}\left(Y_{\sigma}\right)$ ). This shows that the face and degeneracy operators, if they exist, are uniquely determined by the conditions above. To show existence we may suppose $\mathbf{K}=\boldsymbol{\Delta}^{n}$ in which case $Y$ is free and $\operatorname{Hom}_{R}(Y, M)$ is the local system $\Pi_{\alpha}\left\{x_{\alpha}\right\} \times M$. In particular this system is extendable if $M$ is.

(ii) This is true by definition.

Remark 1.11. Given any two $R$-modules $N, M$ we could define a local system of $\mathbb{Z}$-modules $\operatorname{Hom}_{R}(N, M)$ by setting $\operatorname{Hom}_{R}(N, M)_{\sigma}=R^{\sigma}-\operatorname{lin}\left(N^{\sigma}, M^{\sigma}\right)$, and this coincides with the definition in Lemma 1.10 when $N$ is locally free. Note that if the rings $R_{\sigma}$ are commutative, then $\operatorname{Hom}_{R}(M, N)$ is naturally an $R$-module.

Next let $V$ be an $R$-module and $\sigma: \boldsymbol{\Delta}^{n} \rightarrow \mathbf{K}$ be an $n$-simplex and pull $V$ and $R$ back to $V^{\sigma}$ and $R^{\sigma}$ over $\boldsymbol{\Delta}^{n}$. Passing to global sections we have the submodule $V_{\sigma, \partial \sigma} \subset V_{\sigma}$ and the ideal $R_{\sigma, \partial \sigma} \subset R_{\sigma}$, each consisting of the elements vanishing on $\partial \boldsymbol{\Delta}^{n}$.

Lemma 1.12. If $V$ is a locally free $R$-module, then

(i) $V_{\sigma, \partial \sigma}=R_{\sigma, \partial \sigma} V_{\sigma}, \sigma \in \mathbf{K}$.

(ii) $V$ is extendable if $R$ is. 
Proof. (i) Without loss of generality (via pull-back to $\boldsymbol{\Delta}^{n}$ ) we may suppose $\mathbf{K}=$ $\boldsymbol{\Delta}^{n}, \sigma=i d: \boldsymbol{\Delta}^{n} \rightarrow \boldsymbol{\Delta}^{n}$ and $V$ is a direct sum of copies of $R$. Since $\partial \boldsymbol{\Delta}^{n}$ is finite, Lemma 1.7]implies that the functor $\Gamma$ commutes with (possibly infinite) direct sums over $\partial \boldsymbol{\Delta}^{n}$. This reduces us to the trivial case $V=R$.

(ii) is obvious.

In this and the following sections we shall frequently need to lift $R$-linear maps from locally free $R$-modules in analogy with the lift of maps from projective modules through surjections. The appropriate notion replacing surjection in our context is given by

Definition 1.13. An $R$-linear map $\rho: U \rightarrow W$ will be called strongly surjective if $\rho_{v}$ is surjective for each vertex $v \in \mathbf{K}$ and if the $R_{\sigma}$-maps (res, $\left.\rho_{\sigma}\right): U_{\sigma} \rightarrow$ $U_{\partial \sigma} \times_{W_{\partial \sigma}} W_{\sigma}$ are surjective for each $\sigma \in \mathbf{K}_{n}, n \geq 1$.

Proposition 1.14. Let $\rho: U \rightarrow W$ be an R-linear map with $U$ extendable and each $\rho_{\sigma}$ surjective. Then the following conditions are equivalent:

(i) $\rho$ is strongly surjective;

(ii) $\rho$ induces surjections $U_{\sigma, \partial \sigma} \rightarrow W_{\sigma, \partial \sigma}, \sigma \in \mathbf{K}$;

(iii) $\operatorname{ker} \rho$ is extendable.

In particular, if $W$ is locally free, then $\rho$ is strongly surjective.

Proof. The equivalence of (i)-(iii) is straightforward. If $W$ is locally free, then (ii) follows from Lemma 1.12 and the surjectivity of each $\rho_{\sigma}$.

Lemma 1.15. (i) Strong surjectivity inherits to pull-backs.

(ii) If $\rho: U \rightarrow W$ is strongly surjective, then $\Gamma(\rho)$ and each $\rho_{\sigma}$ are surjective.

Proof. (i) This is obvious.

(ii) To show $\Gamma(\rho)$ is surjective, consider some $\Psi \in \Gamma(W)$ and suppose by induction that $\Phi \in \Gamma\left(U_{\mid \mathbf{K}^{(n-1)}}\right)$ satisfies $\Gamma(\rho) \Phi=\Psi_{\mid \mathbf{K}^{(n-1)}}$. It is sufficient to extend $\Phi$ to the $n$-skeleton. For each non-degenerate $n$ simplex $\sigma$ choose $\Phi_{\sigma} \in U_{\sigma}$ so that (res, $\left.\rho_{\sigma}\right) \Phi_{\sigma}=\left(\Phi_{\partial \sigma}, \Psi_{\sigma}\right)$, where $\Phi_{\partial \sigma}$ is the pull-back of $\Phi$ to $\partial \boldsymbol{\Delta}^{n}$. Then apply Remark 1.3 to extend to the degenerate simplices.

Next since strong surjectivity inherits to pull-backs, what we have just showed proves the surjectivity of $U_{\partial \sigma} \rightarrow W_{\partial \sigma}$ for all simplices of $\mathbf{K}$. Thus $U_{\partial \sigma} \times{ }_{W_{\partial \sigma}} W_{\sigma} \rightarrow$ $W_{\sigma}$ is surjective and $\rho_{\sigma}$ is the composite of this with the surjection (res, $\rho_{\sigma}$ ).

Proposition 1.16. Suppose given an R-linear diagram

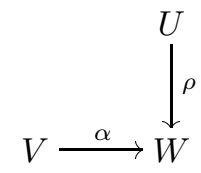

in which $\rho$ is strongly surjective. If each $V_{\sigma}$ is $R_{\sigma}$-projective, then there is an $R$-linear map $\beta: V \rightarrow U$ such that $\rho \beta=\alpha$. (We call $\beta$ a lift of $\alpha$.)

Proof. We suppose by induction that $\beta$ has been constructed over the $k$ simplices of $\mathbf{K}, k<n$, compatible with face operators and degeneracy maps where defined. 
Let $\sigma$ be any $n$-simplex and note that we have a diagram of $R_{\sigma}$-modules

$$
V_{\sigma} \underset{\left(\Gamma(\beta) \text { ores }, \alpha_{\sigma}\right)}{\longrightarrow} U_{\partial \sigma} \perp_{W_{\partial \sigma}}^{U_{\sigma}} W_{\sigma}
$$

By hypothesis, (res, $\left.\rho_{\sigma}\right)$ is surjective and $V_{\sigma}$ is $R_{\sigma}$-projective. Lift $\left(\Gamma(\beta) \circ\right.$ res, $\left.\alpha_{\sigma}\right)$ over (res, $\rho_{\sigma}$ ) to obtain an $R_{\sigma}$-linear map $\gamma_{\sigma}: V_{\sigma} \rightarrow U_{\sigma}$ satisfying $\rho_{\sigma} \gamma_{\sigma}=\alpha_{\sigma}$ and $\partial_{i} \gamma_{\sigma}=\gamma_{\partial_{i} \sigma}, 1 \leq i \leq n$. If $\sigma$ is non-degenerate, we set $\beta_{\sigma}=\gamma_{\sigma}$. If $\sigma$ is degenerate, let $I=\left\{i \mid \sigma=s_{i} \tau_{i}\right\}$. Note that $\tau_{i}=\partial_{i} \sigma$.

Without loss of generality, and to simplify the notation, we shall suppose $I=$ $\{0,1, \ldots, k\}$ and we set $J=\{k+1, \ldots, n-1\}$.

Let $S_{\sigma} \subset V_{\sigma}$ be the $R_{\sigma}$-submodule generated by the $s_{i}\left(V_{\tau_{i}}\right), i \in I$. We show that

$$
V_{\sigma}=S_{\sigma} \oplus \bigcap_{i=0}^{k} k e r \partial_{i}
$$

and that a map $\theta: S_{\sigma} \rightarrow U_{\sigma}$ is defined by

$$
\theta: \sum s_{i} x_{i} \mapsto \sum s_{i} \beta_{\tau_{i}}\left(x_{i}\right), \quad x_{i} \in V_{\tau_{i}} .
$$

Then we define $\beta_{\sigma}$ to be $\theta$ in $S_{\sigma}$ and $\gamma_{\sigma}$ in $\bigcap_{i \in I} \operatorname{ker} \partial_{i}$. To establish (1.17) and (1.18) we first observe that a relation of the form $\partial_{0}\left(s_{0} x_{0}+\cdots+s_{k} x_{k}\right)=0$ implies that $x_{0}=-\sum_{j=1}^{k} s_{j-1} \partial_{0} x_{j}$, whence $s_{0} x_{0}=-\sum_{j=1}^{k} s_{j} s_{0} \partial_{0} x_{j}$.

We now prove (1.17). For any $v \in V_{\sigma}$, define elements $v_{i} \in V_{\sigma}$ inductively by $v_{0}=$ $v$, and $v_{i+1}=v_{i}-s_{i} \partial_{i} v_{i}$. Then $v_{i+1} \in \bigcap_{j \leq i}$ ker $\partial_{j}$. Clearly $v=\sum_{i=0}^{k} s_{i} \partial_{i} v_{i}+v_{k+1}$, which establishes that $V_{\sigma}=S_{\sigma}+\bigcap_{i=0}^{k} k e r \partial_{i}$. On the other hand if $v=\sum_{i=0}^{k} s_{i} x_{i} \in$ $\bigcap_{i=0}^{k}$ ker $\partial_{i}$ we have by the remarks above that

$$
v=\sum_{j=1}^{k} s_{j}\left(x_{j}-s_{0} \partial_{0} x_{j}\right) \in \bigcap_{i=1}^{k} \operatorname{ker} \partial_{i} .
$$

Iterating this procedure we find $v=s_{k} x$ with $0=\partial_{k} v=x$, i.e., $v=0$.

The same type of argument shows that $\theta$ is well defined.

\section{2. $R$ MINIMAL MODELS}

In this section, $\mathbf{K}$ is a connected simplicial set, $\mathbf{k}$ is a field of characteristic zero and $R$ is an extendable local system of commutative $\mathbf{k}$-algebras on $\mathbf{K}$. We consider $R$ as a graded object, concentrated in degree zero.

Recall (Definition 1.5) that an $R$-CDGA is a local system of commutative differential graded algebras over $R$. In this section we extend Sullivan's theory of minimal models to this context. For simplicity we shall restrict ourselves to the 1-connected case, but the results in this section can be extended to the general situation. Recall that if $Z=\left\{Z_{i}\right\}_{i \geq 1}$ is a graded $\mathbf{k}$-vector space, then

$$
\Lambda Z=\text { exterior algebra }\left(Z^{\text {odd }}\right) \otimes \operatorname{symmetric} \text { algebra }\left(Z^{\text {even }}\right)
$$

is the free graded commutative k-algebra on $Z$. A 1-connected Sullivan algebra (called KS-complex in [13]) is a CDGA of the form $(\Lambda Z, d)$ in which $Z$ is the increasing union of subspaces $0=Z(-1) \subset Z(0) \subset Z(1) \subset \cdots \subset Z(j) \subset \cdots$ with 
$d Z(j) \subset \Lambda Z(j-1)$. If $\operatorname{Im} d \subset(\Lambda Z)^{+} \cdot(\Lambda Z)^{+}$, then $(\Lambda Z, d)$ is called minimal. In this case $d: Z^{n} \rightarrow \Lambda Z^{<n}$. We say that $(\Lambda Z, d)$ has finite type if each $Z^{i}$ is finite dimensional. For the theory we refer to [21], [13, [1].

Now we set some notation. If $A$ is a local system of $\mathbf{k}$-CDGA's, we define a local system $R \otimes_{\mathbf{k}} A$ of $R$-CDGA's, by $\left(R \otimes_{\mathbf{k}} A\right)_{\sigma}=R_{\sigma} \otimes_{\mathbf{k}} A_{\sigma}$. Given a local system of graded k-vector spaces $Z$ we define the local system $\Lambda Z$ by $(\Lambda Z)_{\sigma}=\Lambda Z_{\sigma}$. Similarly we set $\otimes Z=\left\{\otimes Z_{\sigma}\right\}, \otimes Z_{\sigma}$ denoting the tensor algebra on $Z_{\sigma}$.

Lemma 2.1. If $V$ is an extendable local system of graded $\mathbf{k}$-vector spaces, then so is $\Lambda V$.

Proof. Since $\mathbb{Q} \subset \mathbf{k}$ the projection $\pi: \otimes V \rightarrow \Lambda V$ restricts to an isomorphism from the submodule of graded skew symmetric tensors on $V$. Thus $\otimes V \cong k e r \pi \oplus \Lambda V$. Since the tensor product over $\mathbf{k}$ of extendable local systems is extendable 13 . Theorem 12.37], the lemma follows from Lemma 1.8 .

Given a graded $R$-module $Y$ we define the local system $\Lambda Y$ by $(\Lambda Y)_{\sigma}=\Lambda Y_{\sigma}$, $\Lambda Y_{\sigma}$ denoting the free commutative graded $R_{\sigma}$-algebra on $Y_{\sigma}$. Thus if $Y=R \otimes_{\mathbf{k}} Z$, then $\Lambda Y=R \otimes_{\mathbf{k}} \Lambda Z$.

Definition 2.2. (1) A local system of k-CDGA's (resp. an $R$-CDGA), $A$, is 1connected if $H^{0}(A)$ is the constant local system $\mathbf{k}$ (resp. if $H^{0}(A)=R$ ) and if $H^{1}(A)=0$.

(2) A 1-connected $R$ minimal model is an $R$-CDGA, $M$, such that, for some fixed 1-connected minimal Sullivan algebra, $(\Lambda Z, d)$, and for each $\sigma \in \mathbf{K}$, there is an isomorphism of $R^{\sigma}$-CDGA's: $M^{\sigma} \cong R^{\sigma} \otimes_{\mathbf{k}}(\Lambda Z, d)$. We call $(\Lambda Z, d)$ a representative Sullivan algebra for $M$. (We shall see in Proposition 2.5 that $M$ has the form $(\Lambda Y, d)$.

Our main result establishes the existence and uniqueness of models in certain cases:

Theorem 2.3. Let $A$ be a 1-connected extendable local system of $\mathbf{k}-C D G A$ 's on $\mathbf{K}$ such that the system $H(A)$ is locally constant. Then there exists a 1-connected $R$ minimal model, $(\Lambda Y, d)$, and a quasi-isomorphism of $R$-CDGA's, $m:(\Lambda Y, d) \stackrel{\simeq}{\rightarrow}$ $R \otimes_{\mathbf{k}}$ A. Moreover $(\Lambda Y, d)$ is uniquely determined up to isomorphism.

Definition 2.4. The morphism $m:(\Lambda Y, d) \rightarrow R \otimes_{\mathbf{k}} A$ is called an $R$ minimal model for $R \otimes_{\mathbf{k}} A$.

The proof of the theorem requires three preliminary propositions.

Proposition 2.5. Let $M$ be a 1-connected $R$ minimal model. Then

(i) There exists an isomorphism of local systems of commutative graded algebras $M \cong \Lambda Y$ with $Y=\left\{Y^{i}\right\}_{i>2}$ and each $Y^{i}$ locally free (Definition 1.9). In particular, $M$ is locally free and hence extendable.

(ii) The differential $d$ of the local system $\Lambda Y$ restricts to $R$-linear maps: $d$ : $Y^{i} \rightarrow \Lambda Y^{<i}$. 
Proposition 2.6. Let $(\Lambda Y, d)$ be a 1-connected $R$ minimal model and suppose given a diagram of $R-C D G A$ 's

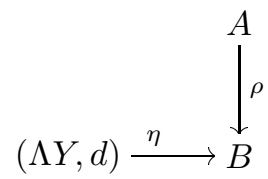

Then there is an $R$-CDGA morphism $\varphi:(\Lambda Y, d) \rightarrow A$ such that $\rho \varphi=\eta$, provided that, either

(i) $A$ is extendable and $\rho$ is a strongly surjective quasi-isomorphism, or

(ii) $\mathbf{K}=\boldsymbol{\Delta}^{n}$ and $\Gamma(\rho)$ is a surjective quasi-isomorphism.

Before stating the third proposition we introduce a little more notation. If $M$ is a local system of $R$-CDGA's, with $M^{0}=R$, we define local systems $M^{+}$, $M^{+} \cdot M^{+}$and $Q(M)$ by: $\left(M^{+}\right)_{\sigma}=M_{\sigma}^{>0},\left(M^{+} \cdot M^{+}\right)_{\sigma}=M_{\sigma}^{+} \cdot M_{\sigma}^{+}$and $Q(M)=$ $M^{+} /\left(M^{+} \cdot M^{+}\right)$. In particular, if $(\Lambda Y, d)$ is a 1-connected $R$ minimal model (2.2), one has $Q(\Lambda Y) \cong Y$. Moreover since $d(\Lambda Y) \subset(\Lambda Y)^{+} \cdot(\Lambda Y)^{+}$, the natural $R$-linear surjection $(\Lambda Y)^{+} \rightarrow Q(\Lambda Y)$ induces an $R$-linear map

$$
H^{+}(\Lambda Y) \stackrel{\zeta}{\rightarrow} Q(\Lambda Y) \text {. }
$$

Similarly, $d:(\Lambda Y)^{n} \rightarrow \Lambda Y^{<n}$ factors over (2.7) to yield an $R$-linear map $Q^{n}(\Lambda Y)$ $\stackrel{\delta}{\rightarrow} H^{n+1}\left(\Lambda Y^{<n}\right)$. Finally, the sequence

$$
H^{n}\left(\Lambda Y^{<n}\right) \rightarrow H^{n}(\Lambda Y) \stackrel{\zeta}{\rightarrow} Q^{n}(\Lambda Y) \stackrel{\delta}{\rightarrow} H^{n+1}\left(\Lambda Y^{<n}\right) \rightarrow H^{n+1}(\Lambda Y)
$$

is naturally identified with part of the long exact sequence associated to $\Lambda Y^{<n} \rightarrow$ $\Lambda Y \rightarrow \Lambda Y / \Lambda Y^{<n}$.

Proposition 2.9. Let $\varphi: \Lambda Y \rightarrow \Lambda Z$ be a morphism between 1-connected $R$ minimal models. The following conditions are then equivalent:

(i) $H(\varphi)$ is an isomorphism.

(ii) $Q(\varphi)$ is an isomorphism.

(iii) $\varphi$ is an isomorphism.

Proof of 2.5. (i) We wish to apply Proposition 1.16 to the quotient map $\rho: M^{+} \rightarrow$ $Q(M)$. It is immediate from Definition 2.2 that each $\rho_{\sigma}$ is surjective and that $M, M^{+}, M^{+} \cdot M^{+}$and $Q(M)$ are locally free (1.9). Now the final assertion of Proposition 1.14implies that $\rho$ is strongly surjective and we may apply Proposition 1.16. It gives a section $\beta: Q(M) \rightarrow M^{+}$such that $\rho \beta=$ id. Extend $\beta$ to a morphism of local systems of algebras: $\Lambda Q(M) \rightarrow M$. Our hypothesis implies that this pulls back to isomorphisms $\Lambda Q(M)^{\sigma} \cong M^{\sigma}$. Hence it is an isomorphism. Thus $M$ is locally free, and extendable by Lemma 1.12 (ii).

(ii) It is sufficient to check this on the pull-backs $M^{\sigma}$, where it is true by definition.

Proof of 2.6. (i) We assume $\varphi$ is constructed on the simplices of dimension less than $n$, compatible with the face operators and the degeneracy maps where defined, and show we can extend $\varphi$ to the $n$ simplices with the same compatibility.

First consider a non-degenerate $n$-simplex $\sigma$. To extend $\varphi$ to $\sigma$ we may work over the pull-backs $(-)^{\sigma}$; thus we are reduced to the case $\mathbf{K}=\boldsymbol{\Delta}^{n}$ and $\sigma=i d$ : 
$\boldsymbol{\Delta}^{n} \rightarrow \boldsymbol{\Delta}^{n}$. In particular, we may write $(\Lambda Y, d)=R \otimes_{\mathbf{k}}(\Lambda Z, d)$ where $(\Lambda Z, d)$ is a classical minimal Sullivan algebra.

Since we are already given $\varphi$ on the faces of $\sigma$, we obtain the commutative diagram of CDGA morphisms

$$
(\Lambda Z, d) \underset{\left(\varphi_{\partial \sigma}, \eta_{\sigma}\right)}{\longrightarrow} A_{\partial \sigma} \times_{B_{\partial \sigma}}^{A_{\sigma}} B_{\sigma}
$$

By the hypothesis of strong surjectivity, (res, $\rho_{\sigma}$ ) is surjective. We shall show it is a quasi-isomorphism. Since the kernel of $\left(\right.$ res, $\left.\rho_{\sigma}\right)$ is $(k e r \rho)_{\sigma, \partial \sigma}$, we have precisely to show that

$$
H\left((\operatorname{ker} \rho)_{\sigma, \partial \sigma}\right)=0 .
$$

For this, observe first that since $A$ is extendable and $\rho$ is strongly surjective, $\operatorname{ker} \rho$ is extendable (Proposition 1.14). Thus $(k e r \rho)_{\sigma, \partial \sigma}$ fits in the short exact sequence

$$
0 \rightarrow(\operatorname{ker} \rho)_{\sigma, \partial \sigma} \rightarrow(\operatorname{ker} \rho)_{\sigma} \rightarrow(\operatorname{ker} \rho)_{\partial \sigma} \rightarrow 0 .
$$

We establish (2.10) by proving that $H\left((\operatorname{ker} \rho)_{\sigma}\right)=0=H\left((\operatorname{ker} \rho)_{\partial \sigma}\right)$. Now for all $\tau, \rho_{\tau}$ is a strongly surjective quasi-isomorphism. Thus $\rho_{\tau}$ is also surjective (Lemma 1.15) and $H\left(k e r \rho_{\tau}\right)=0$. In particular, $0 \rightarrow \operatorname{ker} \rho$ is a quasi-isomorphism between extendable local systems. Hence by [13, Theorem 12.27], for all $\mathbf{L} \subset \boldsymbol{\Delta}^{n}$, $H\left(\Gamma\left(k e r \rho_{\mid \mathbf{L}}\right)\right)=0$. In particular, $H(k e r \rho)_{\partial \sigma}=0$, and (2.10) is established.

Thus (res, $\rho_{\sigma}$ ) is a surjective quasi-isomorphism and the classical lifting theorem for Sullivan models 21, Corollary 3.6], 13, Proof of Theorem 5.19] gives a CDGA morphism $\varphi_{\sigma}:(\Lambda Z, d) \rightarrow A_{\sigma}$ extending $\varphi_{\partial \sigma}$, and such that $\rho_{\sigma} \varphi_{\sigma}=\eta_{\sigma}$.

Suppose now that $\sigma$ is degenerate and write $\sigma=s_{i} \tau$. Then pull-back all the local systems over $\tau$ to $\boldsymbol{\Delta}^{n-1}$, and again write $(\Lambda Y, d)=R \otimes_{\mathbf{k}}(\Lambda Z, d)$. By hypothesis we are given $\varphi_{\tau}:(\Lambda Z, d) \rightarrow A_{\tau}$, and now we define $\varphi_{\sigma}$ by $\varphi_{\sigma}=s_{i} \circ \varphi_{\tau}:(\Lambda Z, d) \rightarrow A_{\sigma}$. It is easy to see that this is independent of the choice of $s_{i}$ and satisfies the desired compatibility conditions.

(ii) Since $\mathbf{K}=\boldsymbol{\Delta}^{n}$, we may write $(\Lambda Y, d)=R \otimes_{\mathbf{k}}(\Lambda Z, d)$, where $(\Lambda Z, d)$ is a classical minimal Sullivan algebra. The CDGA morphism $\Gamma(\eta)$ restricts to a morphism $(\Lambda Z, d) \rightarrow \Gamma(B)$, which lifts through $\Gamma(\rho)$ to a morphism $\Phi:(\Lambda Z, d) \rightarrow$ $\Gamma(A)$. Define $\varphi$ by $\varphi_{\sigma}(r \otimes w)=r . \Phi(w)_{\sigma}$.

Proof of 2.9. The assertions (ii) $\Rightarrow$ (iii) and (iii) $\Rightarrow$ (i) are obvious, and (i) $\Rightarrow$ (ii) follows directly from (2.8) and an inductive argument.

Proof of Theorem 2.3. Since $\mathbf{K}$ is connected, our hypothesis on $H(A)$ implies that all the CDGA's $A_{\sigma}$ are connected by chains of CDGA quasi-isomorphisms. Hence we can find a single 1-connected minimal Sullivan algebra $(\Lambda Z, d)$, in the classical sense, and for each $\sigma \in \mathbf{K}$ a CDGA quasi-isomorphism $(\Lambda Z, d) \stackrel{\simeq}{\rightrightarrows} A_{\sigma}$. Next, we choose a CDGA surjection $N_{\sigma} \rightarrow A_{\sigma}$ in which $\varepsilon_{\sigma}: N_{\sigma} \rightarrow \mathbf{k}$ is an augmented CDGA satisfying $H\left(N_{\sigma}\right)=\mathbf{k}$. Tensoring these morphisms together yields a surjective CDGA quasi-isomorphism $\alpha_{\sigma}:(\Lambda Z, d) \otimes_{\mathbf{k}} N_{\sigma} \stackrel{\simeq}{\rightarrow} A_{\sigma}$. This induces a morphism $\hat{\alpha}_{\sigma}$ from the constant local system $(\Lambda Z, d) \otimes_{\mathbf{k}} N_{\sigma}$ on $\boldsymbol{\Delta}^{|\sigma|}$ to $A^{\sigma}$, which extends to $\beta_{\sigma}=R^{\sigma} \otimes_{\mathbf{k}} \hat{\alpha}_{\sigma}: R^{\sigma} \otimes_{\mathbf{k}}(\Lambda Z, d) \otimes_{\mathbf{k}} N_{\sigma} \rightarrow R^{\sigma} \otimes_{\mathbf{k}} A^{\sigma}$. We may identify $\Gamma\left(\beta_{\sigma}\right)=$ 
$R_{\sigma} \otimes \alpha_{\sigma}$, so that $\Gamma\left(\beta_{\sigma}\right)$ is also a surjective quasi-isomorphism. On the other hand since $H(A)$ is locally constant, $\beta_{\sigma}$ is a quasi-isomorphism of local systems.

We are now ready to construct the desired $(\Lambda Y, d)$ and quasi-isomorphism $(\Lambda Y, d)$ $\rightarrow R \otimes_{\mathbf{k}} A$, by induction on degree. More precisely we suppose constructed:

- For each $i, 2 \leq i<n$, locally free $R$-modules, $Y^{i}$. We write $Y^{<n}=\left\{Y^{i}\right\}_{2<i<n}$.

- An $R$-CDGA of the form $\left(\Lambda Y^{<n}, d\right)$ and a morphism $\psi:\left(\Lambda Y^{<n}, d\right) \rightarrow R \otimes_{\mathbf{k}} A$.

- For each $\sigma \in \mathbf{K}$ a morphism $\varphi_{\sigma}:\left(\Lambda Y^{<n}, d\right)^{\sigma} \rightarrow R^{\sigma} \otimes_{\mathbf{k}}(\Lambda Z, d) \otimes_{\mathbf{k}} N_{\sigma}$ such that

(a) the diagram

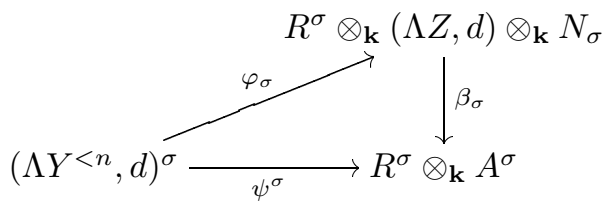

commutes and

(b) the composite (id $\left.\otimes \varepsilon_{\sigma}\right) \circ \varphi_{\sigma}$ defines an isomorphism:

$$
\left(\mathrm{id} \otimes \varepsilon_{\sigma}\right) \circ \varphi_{\sigma}:\left(\Lambda Y^{<n}, d\right)^{\sigma} \stackrel{\cong}{\rightrightarrows} R^{\sigma} \otimes_{\mathbf{k}}\left(\Lambda Z^{<n}, d\right) .
$$

We show that we can extend the construction to $i=n$. First, we recall that any chain complex morphism $f: C^{\prime} \rightarrow C$ is naturally equivalent to the inclusion in the short exact sequence $0 \rightarrow C^{\prime} \rightarrow C^{\prime} \oplus C \oplus s C^{\prime} \rightarrow C \oplus s C^{\prime} \rightarrow 0$, in which in the central term $\left(s C^{\prime}\right)^{i}=\left(C^{\prime}\right)^{i+1}$ and $d s x=-x+f(x)-s d x$. The quotient $C \oplus s C^{\prime}$ is called the cofibre, $C(f)$, of $f$ and its homology fits in the natural long cofibre sequence: $\ldots \rightarrow H\left(C^{\prime}\right) \rightarrow H(C) \rightarrow H(C(f)) \stackrel{\delta}{\rightarrow} H\left(C^{\prime}\right) \rightarrow \ldots$

Because these constructions are natural, they also apply to local systems. In particular, let $C(\psi)$ be the cofibre of the morphism $\psi:\left(\Lambda Y^{<n}, d\right) \rightarrow R \otimes_{\mathbf{k}} A$. Clearly cofibres commute with pull-backs, so we can identify $(C(\psi))^{\sigma}=C\left(\psi^{\sigma}\right)$. On the other hand $i d \otimes \varepsilon_{\sigma}$ and the quasi-isomorphism $\beta_{\sigma}$ identify the cofibre sequence for $\psi^{\sigma}$ with that for the inclusion

$$
R^{\sigma} \otimes_{\mathbf{k}}\left(\Lambda Z^{<n}, d\right) \rightarrow R^{\sigma} \otimes_{\mathbf{k}}(\Lambda Z, d) .
$$

In the case of an inclusion $C^{\prime} \rightarrow C$ of chain complexes, however, the cofibre sequence is just the long exact homology sequence associated to the short exact sequence $0 \rightarrow C^{\prime} \rightarrow C \rightarrow C / C^{\prime} \rightarrow 0$. We have at once that $H^{i}(C(\psi))=0, i<n$. Moreover, (2.8) yields a commutative diagram of $R^{\sigma}$-linear maps

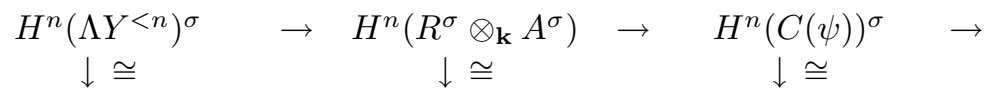

$$
\begin{aligned}
& R^{\sigma} \otimes_{\mathbf{k}} H^{n}\left(\Lambda Z^{<n}\right) \quad \rightarrow \quad R^{\sigma} \otimes_{\mathbf{k}} H^{n}(\Lambda Z) \rightarrow R^{\sigma} \otimes_{\mathbf{k}} Q^{n}(\Lambda Z) \rightarrow
\end{aligned}
$$

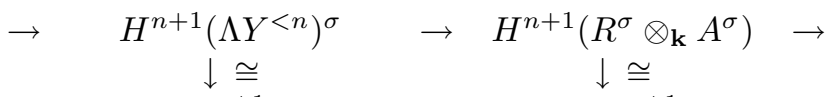

$$
\begin{aligned}
& \rightarrow R^{\sigma} \otimes_{\mathbf{k}} H^{n+1}\left(\Lambda Z^{<n}\right) \rightarrow R^{\sigma} \otimes_{\mathbf{k}} H^{n+1}(\Lambda Z) \rightarrow
\end{aligned}
$$

in which the upper row is the pull-back of the cofibre sequence for $\psi$.

This diagram shows that the $R$-module $H^{n}(C(\psi))$ is locally free. The isomorphism (b) of our induction hypothesis identifies the surjection $\xi:($ ker $d) \cap$ $\Lambda Y^{<n} \rightarrow H\left(\Lambda Y^{<n}\right)$ as a surjection between locally free $R$-modules. Hence by 
Proposition 1.14, $\xi$ is strongly surjective and so by Proposition 1.16 the connecting homomorphism $\delta: H^{n}(C(\psi)) \rightarrow H^{n+1}\left(\Lambda Y^{<n}\right)$ lifts through $\xi$ to an $R$ linear map: $\theta: H^{n}(C(\psi)) \rightarrow($ ker $d) \cap\left(\Lambda Y^{<n}\right)^{n+1}$. We regard $\theta$ as a map $\left(H^{n}(C(\psi)), 0\right) \rightarrow(\Lambda Y \leq n, d)$ of degree 1 between differential $R$-modules. Extend $\left(\Lambda Y^{<n}, d\right)$ to $\left(\Lambda Y^{\leq n}, d\right)$ by setting $Y^{n}=H^{n}(C(\psi))$ and $d=\theta$ in $Y^{n}$.

The composite $H(\psi) \circ H(\theta): Y^{n} \rightarrow H^{n+1}\left(\Lambda Y^{<n}\right) \rightarrow H^{n+1}\left(R \otimes_{\mathbf{k}} A\right)$ is just $H(\psi) \circ \delta=0$, because the cofibre sequence is exact. Thus we can regard $\psi \circ d$ as an $R$-linear map $\psi \circ d: Y^{n} \rightarrow R \otimes_{\mathbf{k}} d\left(A^{n}\right)$. We may apply [13, Lemma 12.48 (i)] to conclude that the kernel of the surjective morphism $i d \otimes d: R \otimes_{\mathbf{k}} A \rightarrow R \otimes_{\mathbf{k}} d(A)$ is extendable. Since $Y^{n}$ is locally free, we may use Proposition 1.16 to lift $\psi \circ d$ through this morphism to obtain an $R$-linear map $Y^{n} \rightarrow R \otimes_{\mathbf{k}} A^{n}$. This defines a unique extension of $\psi$ to a morphism of $R$-CDGA's $\Psi:(\Lambda Y \leq n, d) \rightarrow R \otimes_{\mathbf{k}} A$.

Finally, recall the quasi-isomorphism $\beta_{\sigma}$. Since $\Gamma\left(\beta_{\sigma}\right)$ is a surjective quasiisomorphism and $Y^{n}$ is locally free, the argument of Proposition 2.6 (ii) shows that we can extend $\varphi_{\sigma}$ to a lift $\Phi_{\sigma}:\left(\Lambda Y^{\leq n}, d\right)^{\sigma} \rightarrow R^{\sigma} \otimes_{\mathbf{k}}(\Lambda Z, d) \otimes_{\mathbf{k}} N_{\sigma}$ of $\Psi_{\sigma}$ over $\beta_{\sigma}$. Consider the composite $\gamma=\left(i d \otimes \varepsilon_{\sigma}\right) \circ \Phi_{\sigma}:\left(\Lambda Y^{\leq n}\right)^{\sigma} \rightarrow R^{\sigma} \otimes_{\mathbf{k}} \Lambda Z$. By construction, $\Psi$ induces an isomorphism $H^{n}\left(\Lambda Y^{\leq n} / \Lambda Y^{<n}\right) \stackrel{\cong}{\rightrightarrows} H^{n}(C(\psi))$. Hence $\gamma$ induces an isomorphism $H^{n}\left(\Lambda Y^{\leq n} / \Lambda Y^{<n}\right)^{\sigma} \stackrel{\cong}{\rightrightarrows} H^{n}\left(R^{\sigma} \otimes_{\mathbf{k}} \Lambda Z / \Lambda Z^{<n}\right)$. By (2.8) this isomorphism can be identified with $Q^{n}(\gamma)$. Since $\gamma$ restricts to an isomorphism $\left(\Lambda Y^{<n}\right)^{\sigma} \stackrel{\cong}{\rightrightarrows} R^{\sigma} \otimes_{\mathbf{k}} \Lambda Z^{<n}$, it follows that $\gamma$ itself is an isomorphism from $\left(\Lambda Y^{\leq n}\right)^{\sigma}$ to $R^{\sigma} \otimes_{\mathbf{k}} \Lambda Z \leq n$. The inductive step is now complete and, with it, the proof of the existence of $(\Lambda Y, d)$ and $m$.

It remains to prove uniqueness. Define a local system $(\mathcal{U}(A), \delta)$ of k-CDGA's by: $\mathcal{U}(A)=\Lambda(A \oplus V) ; \delta: A \stackrel{\cong}{\rightrightarrows} V$. The identity map of $A$ extends to a unique surjective morphism of local systems of k-CDGA's, $\rho:(\mathcal{U}(A), \delta) \rightarrow A$. The identity map also defines a section $\eta: A \rightarrow \mathcal{U}(A)$ in the category of local systems of $\mathbf{k}$-vector spaces, and there is an obvious augmentation $\varepsilon$ from $\mathcal{U}(A)$ to the constant local system $\mathbf{k}$.

Now suppose $m:(\Lambda Y, d) \stackrel{\simeq}{\rightarrow} R \otimes_{\mathbf{k}} A$, and $m^{\prime}:\left(\Lambda Y^{\prime}, d^{\prime}\right) \stackrel{\simeq}{\rightrightarrows} R \otimes_{\mathbf{k}} A$ are 1connected $R$ minimal models for $R \otimes_{\mathbf{k}} A$. Consider the diagram

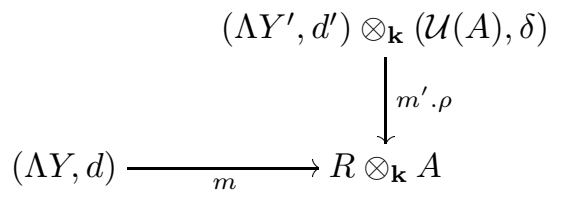

We wish to verify the hypotheses of Proposition 2.6. On the one hand, since $H(\mathcal{U}(A))$ is the constant system $\mathbf{k}$, we deduce that $m^{\prime} . \rho$ is a quasi-isomorphism. On the other, by Lemma 2.1. $\mathcal{U}(A)$ is extendable. Since the tensor product of extendable local systems is extendable [13, Theorem 12.37], it follows that $\Lambda Y^{\prime} \otimes_{\mathbf{k}}$ $\mathcal{U}(A)$ is extendable. Moreover, since $R \otimes_{\mathbf{k}} \eta$ provides an $R$-linear section for $m^{\prime} . \rho$, it follows from Lemma 1.8 that $\operatorname{ker}\left(m^{\prime} . \rho\right)$ is extendable. Since each $\rho_{\sigma}$ is surjective, so is $\left(m^{\prime} . \rho\right)_{\sigma}$ and now Proposition 1.14 asserts that $m^{\prime} . \rho$ is strongly surjective.

We can therefore apply Proposition [2.6 to lift $m$ through $m^{\prime} . \rho$ to a morphism $\omega:(\Lambda Y, d) \rightarrow\left(\Lambda Y^{\prime}, d^{\prime}\right) \otimes_{\mathbf{k}}(\mathcal{U}(A), \delta)$, which will be a quasi-isomorphism because $m$ and $m^{\prime} . \rho$ are. Composing with the quasi-isomorphism $\Lambda Y^{\prime} \otimes_{\mathbf{k}} \varepsilon:\left(\Lambda Y^{\prime}, d^{\prime}\right) \otimes_{\mathbf{k}}$ $(\mathcal{U}(A), \delta) \rightarrow\left(\Lambda Y^{\prime}, d^{\prime}\right)$ yields a quasi-isomorphism $(\Lambda Y, d) \rightarrow\left(\Lambda Y^{\prime}, d^{\prime}\right)$. By Proposition 2.9 this is an isomorphism. 
We complete this section by establishing a result about derivations from $R$ minimal models (Proposition 2.14) that is critical for the sequel.

Let $A$ be a differential graded algebra over a commutative $\operatorname{ring} S$, and let $M$ be a graded differential $A$-bimodule. Recall that a derivation of degree $k$ from $A$ into $M$ is an $S$-linear map $\theta: A^{*} \rightarrow M^{*+k}$ such that $\theta(a b)=\theta(a) \cdot b+(-1)^{\operatorname{deg} a \operatorname{deg} \theta} a \cdot \theta(b)$. Thus the derivations form a graded differential $S$-module $\operatorname{Der}_{S}(A, M)$ with differential given by $d(\theta)=d \circ \theta-(-1)^{\operatorname{deg} \theta} \theta \circ d$.

Definition 2.12. (1) A bimodule over an $R$ minimal model $(\Lambda Y, D)$ is a graded differential $R$-module, $M$, together with a $(\Lambda Y, D)_{\sigma}$-bimodule structure in each $M_{\sigma}$, compatible with the face and degeneracy operators. A morphism of bimodules is an $R$-linear map, commuting with the differentials and preserving the bimodule structure.

(2) A derivation from $(\Lambda Y, D)$ to a bimodule $M$ is an $R$-linear map $\theta: \Lambda Y \rightarrow M$ of some degree, such that each $\theta_{\sigma}$ is a derivation from $(\Lambda Y)_{\sigma} \rightarrow M_{\sigma}$. The graded differential $\Gamma(R)$-module of all such derivations is denoted by $\operatorname{Der}_{R}(\Lambda Y, M)$.

(3) Given a morphism $\varphi: M \rightarrow N$ of $(\Lambda Y, D)$-bimodules we define $\operatorname{Der}_{R}(\Lambda Y, \varphi)$ : $\operatorname{Der}_{R}(\Lambda Y, M) \rightarrow \operatorname{Der}_{R}(\Lambda Y, N)$ to be the morphism of $\Gamma(R)$ graded differential modules given by $\theta \mapsto \varphi \circ \theta$.

Lemma 2.13. Let $M$ be an extendable bimodule over the $R$ minimal model $(\Lambda Y, D)$. Then

(i) an extendable $R$-module $\operatorname{DER}_{R}(\Lambda Y, M)$ is uniquely determined by the conditions: $\left[\operatorname{DER}_{R}(\Lambda Y, M)\right]_{\sigma}=\operatorname{Der}_{R_{\sigma}}\left((\Lambda Y)_{\sigma}, M_{\sigma}\right) ; \partial_{i}\left(\theta_{\sigma}\right) \circ \partial_{i}=\partial_{i} \circ \theta_{\sigma} ; s_{j}\left(\theta_{\sigma}\right) \circ s_{j}=$ $s_{j} \circ \theta_{\sigma}$.

(ii) $\operatorname{Der}_{R}(\Lambda Y, M)=\Gamma\left(\operatorname{DER}_{R}(\Lambda Y, M)\right)$.

Proof. It is sufficient to note that the restriction to $Y_{\sigma}$ defines an isomorphism $\operatorname{Der}_{R_{\sigma}}\left((\Lambda Y)_{\sigma}, M_{\sigma}\right) \stackrel{\cong}{\rightrightarrows} \operatorname{Hom}_{R_{\sigma}}\left(Y_{\sigma}, M_{\sigma}\right)$ of $R_{\sigma}$-modules and then to apply Lemma 1.10.

Proposition 2.14. Let $\varphi: M \rightarrow N$ be a quasi-isomorphism of extendable bimodules over the $R$ minimal model $(\Lambda Y, D)$. If $R$ is extendable, then $\operatorname{Der}_{R}(\Lambda Y, \varphi)$ is also a quasi-isomorphism.

Proof. The morphism $\varphi$ defines a morphism of differential graded $R$-modules $\Phi$ : $\operatorname{DER}_{R}(\Lambda Y, M) \rightarrow \operatorname{DER}_{R}(\Lambda Y, N)$, given by $\Phi_{\sigma}\left(\theta_{\sigma}\right)=\varphi_{\sigma} \circ \theta_{\sigma}$. We show first that $\Phi$ is a quasi-isomorphism.

Let $(\Lambda Z, d)$ be a representative Sullivan model for $(\Lambda Y, D)$. Then for each $\sigma$ we can identify $\Phi_{\sigma}$ as the morphism $\Psi_{\sigma}: \operatorname{Der}_{\mathbf{k}}\left(\Lambda Z, M_{\sigma}\right) \rightarrow \operatorname{Der}_{\mathbf{k}}\left(\Lambda Z, N_{\sigma}\right)$, where $\Psi_{\sigma}$ is again the composition with $\varphi_{\sigma}$. Since $\varphi_{\sigma}$ is a quasi-isomorphism, the classical Sullivan theory asserts that so is $\Psi_{\sigma}$. Hence each $\Phi_{\sigma}$ is a quasi-isomorphism.

On the other hand, $\Phi$ is a morphism between extendable $R$-modules (Lemma 2.13). Thus [13, Theorem 12.27] asserts that $\Gamma(\Phi)$ is a quasi-isomorphism. But clearly $\Gamma(\Phi)=\operatorname{Der}_{R}(\Lambda Y, \varphi)$, and so the proof is complete.

\section{A minimal models}

In this section, we work over a field $\mathbf{k}$ of characteristic zero and with the category of connected simplicial sets, $\mathbf{K}$.

A simplicial CDGA, $\left(A(*), \partial_{i}, s_{j}\right)$, determines the local system $A_{\mathbf{K}}$ of CDGA's on $\mathbf{K}$ which assigns to each $\sigma \in \mathbf{K}_{n}$ the CDGA $A(n)$ and with face and degeneracy 
morphisms the $\partial_{i}$ and $s_{j}$. A simplicial CDGA, $A(*)$, is extendable if $A(n) \rightarrow$ $\Gamma\left(A_{\partial \mathbf{\Delta}^{n}}\right)$ is surjective. (Note that in this case $A_{\mathbf{K}}$ is extendable for all $\mathbf{K}$.) There are two particularly important examples we shall consider: $A_{D R}(*)$ and $A_{P L}(*)$.

$\mathbf{A}_{D R}:(\mathbf{k}=\mathbb{R})$ Here $A_{D R}(n)$ is the CDGA of $C^{\infty}$-differential forms on the Euclidian simplex $\Delta^{n}$. The face and degeneracy maps are the DGA-quasi-isomorphisms $A_{D R}\left(\partial_{i}\right)$ and $A_{D R}\left(s_{j}\right)$ induced by the face and degeneracy maps between the Euclidian simplices. In particular $\left(A_{D R}^{0}\right)_{\sigma}=C^{\infty}\left(\Delta^{|\sigma|}\right)$. The CDGA, $\Gamma\left(A_{D R, \mathbf{K}}\right)$, is the algebra of $C^{\infty}$-differential forms on $\mathbf{K}$.

$\mathbf{A}_{P L}$ : Here $\mathbf{k}$ is arbitrary. Let $A_{P L}(n ; \mathbb{Q}) \subset A_{D R}(n)$ be the rational CDGA generated (over $\mathbb{Q}$ ) by the barycentric coordinate functions $t_{i}$. Explicitly

3.1. $A_{P L}(n ; \mathbb{Q})=\Lambda_{\mathbb{Q}}\left(t_{0}, \ldots, t_{n} ; d t_{0}, \ldots, d t_{n}\right) / \sum t_{i}=1, \sum d t_{i}=0$.

Put $A_{P L}(n ; \mathbf{k})=A_{P L}(n ; \mathbb{Q}) \otimes_{\mathbb{Q}} \mathbf{k}$. The maps $A_{D R}\left(\partial_{i}\right)$ and $A_{D R}\left(s_{j}\right)$ restrict to DGA quasi-isomorphisms $A_{P L}(n ; \mathbb{Q}) \leftrightarrows A_{P L}(n-1 ; \mathbb{Q})$ and these extend by $\bigotimes_{\mathbb{Q}} \mathbf{k}$ to the face and degeneracy maps for $A_{P L}(* ; \mathbf{k})$. In particular $\left(A_{P L}^{0}\right)_{\sigma}$ is a polynomial algebra in $\operatorname{dim} \sigma$ variables. The CDGA, $\Gamma\left(A_{P L, \mathbf{K}}\right)$, is Sullivan's algebra of polynomial differential forms on $\mathbf{K}$ with coefficients in $\mathbf{k}$.

Definition 3.2. A simplicial CDGA, $A(*)$, is admissible if it satisfies the conditions

(i) $A(0)=A(0)^{0}=\mathbf{k}$.

(ii) For each $n$ there are elements $t_{1}, \ldots, t_{n} \in A(n)^{0}$ such that $A(n)=A(n)^{0} \otimes_{\mathbf{k}}$ $\Lambda\left(d t_{1}, \ldots, d t_{n}\right)$.

(iii) The simplicial algebra $H(A)(*)$ satisfies: $H^{i}(A)(*)=0, i \geq 1$, and $H^{0}(A)(*)$ is the constant simplicial algebra $\mathbf{k}$.

(iv) The simplicial algebras $A^{i}(*), i \geq 0$, are extendable.

(v) Let $n$ be an integer. If $f \in A(n)^{0}$ satisfies $d f=f w$, for some $w \in A(n)^{1}$, then either $f=0$ or $f$ is invertible in $A(n)^{0}$.

Lemma 3.3. $A_{P L}(*)$ and $A_{D R}(*)$ are admissible.

Proof. Properties (i) and (ii) are immediate from the definition (cf. 3.1). Property (iii) is in [21, §7] or [13, §13.1], and property (iv) is proved in 21, §1] or [13. Proposition 13.8]. For $A(*)=A_{D R}(*)$, (v) is an obvious property of the solution of a linear differential equation. In the case $A(*)=A_{P L}(*), A(n)^{0}=\mathbf{k}\left[t_{1}, \ldots, t_{n}\right]$. The projection of the equation $d f=w f$ on $d t_{i}$ gives $\frac{\partial f}{\partial t_{i}}=g f$ with $g$ a polynomial. Comparing the degree in $t_{i}$ gives $\frac{\partial f}{\partial t_{i}}=0$. Thus $f$ is constant.

For the rest of this section we fix an admissible simplicial $C D G A, A(*)$, and a connected simplicial set $\mathbf{K}$. We will usually denote the local systems $A_{\mathbf{K}}$ and $A_{\mathbf{K}}^{0}$ simply by $A$ and by $R$.

Definition 3.4. An $A$-algebra is a morphism $j_{E}: A \rightarrow E$ of local systems of CDGA's (on $\mathbf{K}$ ) such that $E$ is extendable and the system $H(E)$ is locally constant. An $A$-morphism is a morphism $\varphi: E \rightarrow E^{\prime}$ such that $\varphi \circ j_{E}=j_{E^{\prime}}$. The category of $A$-algebras and $A$-morphisms is denoted by $\mathcal{A}_{\mathbf{K}}$.

An $A$-algebra $E$ will be called 1-connected (resp. of finite type) if for each $\sigma \in \mathbf{K}$ the algebra $H\left(E_{\sigma}\right)$ is 1-connected (resp. if each $H^{k}\left(E_{\sigma}\right)$ is finite dimensional). The full subcategory of 1-connected $A$-algebras of finite type will be denoted by $\mathcal{A}_{\mathbf{K}}^{f}$.

We come now to the notion of minimal model in this setting. Recall $(\S 2)$ that given a graded $R$-module $Y$ we can form the $R$-algebra $\Lambda Y$. Suppose now that we 
have an $A$-algebra of the form $j: A \longrightarrow\left(A \otimes_{R} \Lambda Y, D\right)$, with $j$ the obvious inclusion. Then $A \otimes_{R} \Lambda Y=\bigoplus_{i \geq 0} A^{i} \otimes_{R} \Lambda Y$, and $A^{0} \otimes_{R} \Lambda Y=R \otimes_{R} \Lambda Y=\Lambda Y$. Thus $D$ decomposes as $D=\sum_{i \geq 0} D_{i}$ with $D_{i}: A^{*} \otimes_{R} \Lambda Y \rightarrow A^{*+i} \otimes_{R} \Lambda Y$. In particular, $\left(\Lambda Y, D_{0}\right)$ is an $R$-CDGA.

Definition 3.5. A 1-connected $A$ minimal model is an $A$-algebra that can be put in the form $A \rightarrow\left(A \otimes_{R} \Lambda Y, D\right)$ as above, such that $\left(\Lambda Y, D_{0}\right)$ is a 1-connected $R$-minimal model. A representative Sullivan algebra for $\left(\Lambda Y, D_{0}\right)$ is called a representative Sullivan algebra for $\left(A \otimes_{R} \Lambda Y, D\right)$.

We will denote by $\mathcal{M}_{\mathbf{K}}$ the full subcategory of $\mathcal{A}_{\mathbf{K}}$ consisting of the 1-connected A minimal models of finite type.

Remark 3.6. Let $E=\left(A \otimes_{R} \Lambda Y, D\right)$ be an $A$ minimal model with representative Sullivan algebra $(\Lambda Z, d)$. Fix a vertex, $v$, of $\mathbf{K}$; then $E_{v} \cong(\Lambda Z, d)$. Since $\mathbf{K}$ is connected and $H(E)$ is locally constant, it follows that $H\left(E_{\sigma}\right) \cong H(\Lambda Z, d), \sigma \in \mathbf{K}$. Since $Z=Z^{\geq 2}$ the $A$-algebra $E$ is 1-connected. Moreover the classical theory of Sullivan algebras implies that $E$ has finite type if and only if each $Z^{k}$ is finite dimensional.

Our first main result deals with morphisms between 1-connected $A$ minimal models, $\Phi:\left(A \otimes_{R} \Lambda Y^{\prime}, D^{\prime}\right) \rightarrow\left(A \otimes_{R} \Lambda Y, D\right)$, and generalizes the classical theorem that a quasi-isomorphism between minimal Sullivan algebras is an isomorphism.

Write $\left(\Lambda Y, D_{0}\right)=F^{1} / F_{0}$, where $A \otimes_{R} \Lambda Y$ is filtered by $F^{p}=A^{\geq p} .\left(A \otimes_{R} \Lambda Y\right)=$ $A^{\geq p} \otimes_{R} \Lambda Y$. Thus $\Phi$ induces a quotient morphism $\varphi:\left(\Lambda Y^{\prime}, D_{0}^{\prime}\right) \longrightarrow\left(\Lambda Y, D_{0}\right)$ and the induced morphism between the associated bigraded local systems has the form $\mathcal{E}(\Phi)=A \otimes_{R} \varphi:\left(A \otimes_{R} \Lambda Y^{\prime}, D_{0}^{\prime}\right) \rightarrow\left(A \otimes_{R} \Lambda Y, D_{0}\right)$.

Theorem 3.7. Let $\Phi:\left(A \otimes_{R} \Lambda Y^{\prime}, D^{\prime}\right) \rightarrow\left(A \otimes_{R} \Lambda Y, D\right)$ be an A-morphism between 1-connected $A$ minimal models of finite type. If any one of $\varphi, \Phi$ or $\mathcal{E}(\Phi)$ is a quasiisomorphism then $\varphi, \Phi$ and $\mathcal{E}(\Phi)$ are all isomorphisms.

Our second main result asserts the local triviality of 1-connected $A$ minimal models

Theorem 3.8. Let $\left(A \otimes_{R} \Lambda Y, D\right)$ be a 1-connected $A$ minimal model of finite type. Then for each simplex $\sigma \in \mathbf{K}_{n}$ there is an $A^{\sigma}$-isomorphism: $A^{\sigma} \otimes_{\mathbf{k}}(\Lambda Z, d) \stackrel{\cong}{\rightrightarrows}$ $\left(A \otimes_{R} \Lambda Y, D\right)^{\sigma}$.

Both Theorems 3.7 and 3.8 are fairly straightforward consequences of

Lemma 3.9. Let $(\Lambda Z, d)$ be a 1-connected minimal Sullivan algebra of finite type and suppose $\Phi: A \otimes_{\mathbf{k}}(\Lambda Z, d) \rightarrow\left(A \otimes_{R} \Lambda Y, D\right)$ is an $A$-quasi-isomorphism of $A$ minimal models. Then $\varphi: R \otimes_{\mathbf{k}}(\Lambda Z, d) \rightarrow\left(\Lambda Y, D_{0}\right)$ is an isomorphism.

Proof. We have to prove that each $\varphi_{\sigma}$ is an isomorphism. Thus we may suppose that $\mathbf{K}=\boldsymbol{\Delta}^{n}$ and $\sigma=i d: \boldsymbol{\Delta}^{n} \rightarrow \boldsymbol{\Delta}^{n}$; in particular $A_{\sigma}=A(n)$. Denote $\Phi_{\sigma}$ and $\varphi_{\sigma}$ by $\Psi$ and $\psi$. Identify $\left(\Lambda Y, D_{0}\right)=R \otimes_{\mathbf{k}}\left(\Lambda Z^{\prime}, d^{\prime}\right)$, where $\left(\Lambda Z^{\prime}, d^{\prime}\right)$ is a representative Sullivan algebra for the $R$-minimal model $\left(\Lambda Y, D_{0}\right)$. Let $v$ be the initial vertex of $\boldsymbol{\Delta}^{n}$. Then $R_{v}=\left(A^{0}\right)_{v}=\mathbf{k}$, so that $\Phi_{v}$ is a quasi-isomorphism from $(\Lambda Z, d)$ to $\left(\Lambda Z^{\prime}, d^{\prime}\right)$. Hence $\Phi_{v}$ is an isomorphism [21, Theorem 2.2].

Identify $(\Lambda Z, d)$ and $\left(\Lambda Z^{\prime}, d^{\prime}\right)$ via $\Phi_{v}$. Thus $\Phi$ is identified with a morphism $\Phi: A \otimes_{\mathbf{k}}(\Lambda Z, d) \rightarrow\left(A \otimes_{\mathbf{k}} \Lambda Z, D\right)$ such that $\Phi_{v}$ is the identity of $(\Lambda Z, d)$. Let 
$\varepsilon: A(n) \rightarrow \mathbf{k}$ be the augmentation corresponding to the inclusion of $v$. Then $\Psi: A(n) \otimes_{\mathbf{k}}(\Lambda Z, d) \rightarrow\left(A(n) \otimes_{\mathbf{k}} \Lambda Z, D\right)$ is a quasi-isomorphism satisfying

$$
\left(\varepsilon \otimes_{\mathbf{k}} i d\right) \circ \Psi=\varepsilon \otimes_{\mathbf{k}} i d .
$$

Write $\Psi=\sum \Psi_{i}$ with $\Psi_{i}: A(n)^{*} \otimes_{\mathbf{k}} \Lambda Z \rightarrow A(n)^{*+i} \otimes_{\mathbf{k}} \Lambda Z$. Then $\Psi_{0}$ is a morphism of graded algebras restricting to

$$
\psi:\left(A(n)^{0} \otimes_{\mathbf{k}} \Lambda Z, i d \otimes d\right) \rightarrow\left(A(n)^{0} \otimes_{\mathbf{k}} \Lambda Z, i d \otimes d\right) .
$$

Write $\Lambda^{j} Z=Z \wedge \cdots \wedge Z$ ( $j$ factors). Then, since $Z=Z^{\geq 2}$, one has:

$$
\left\{\begin{array}{l}
\psi: Z \rightarrow A(n)^{0} \otimes_{\mathbf{k}} \Lambda^{\geq 1} Z, \\
D_{1}: A(n)^{0} \otimes_{\mathbf{k}} Z \rightarrow A(n)^{1} \otimes_{\mathbf{k}} \Lambda^{\geq 1} Z, \\
\Psi_{1}: Z \rightarrow A(n)^{1} \otimes_{\mathbf{k}} \Lambda^{\geq 1} Z .
\end{array}\right.
$$

Let $\psi_{0}: A(n)^{0} \otimes_{\mathbf{k}} Z \longrightarrow A(n)^{0} \otimes_{\mathbf{k}} Z$ be the $A(n)^{0}$-linear map defined by: $\psi(x)-\psi_{0}(x) \in A(n)^{0} \otimes_{\mathbf{k}} \Lambda^{\geq 2} Z, x \in Z$. Similarly, define $D_{1,0}: A(n)^{0} \otimes_{\mathbf{k}} Z \longrightarrow$ $A(n)^{1} \otimes_{\mathbf{k}} Z$ by $D_{1}-D_{1,0}: A(n)^{0} \otimes_{\mathbf{k}} Z \rightarrow A(n)^{1} \otimes_{\mathbf{k}} \Lambda^{\geq 2} Z$. We shall show that $\psi_{0}$ is an isomorphism, which will immediately imply that $\psi$ is one as well.

Since $\Psi$ is a morphism of algebras it follows that

$$
\Psi_{1}\left(x_{1} \wedge \ldots \wedge x_{p}\right)=\sum_{i} \psi\left(x_{1} \wedge \ldots \wedge x_{i-1}\right) \Psi_{1}\left(x_{i}\right) \psi\left(x_{i+1} \wedge \ldots \wedge x_{p}\right), \quad x_{\lambda} \in Z
$$

Thus the relations above imply that $\Psi_{1}: \Lambda^{\geq 2} Z \rightarrow A(n)^{1} \otimes_{\mathbf{k}} \Lambda^{\geq 2} Z$. Since $(\Lambda Z, d)$ is minimal, $\Psi_{1} d x \in A(n)^{1} \otimes_{\mathbf{k}} \Lambda^{\geq 2} Z, x \in Z$. Finally since $D_{0}=i d \otimes_{\mathbf{k}} d$ we know that Im $D_{0} \subset A(n) \otimes_{\mathbf{k}} \Lambda^{\geq 2} Z$. Thus the relation $D \Psi x=\Psi d x$ gives $D_{1,0} \psi_{0} x=0, x \in Z$.

Fix some $m$, and let $\left\{x_{\alpha}\right\}$ be a (finite) basis of $Z^{m}$. Then $\psi_{0}\left(x_{\alpha}\right)=\sum_{\beta} f_{\alpha \beta} x_{\beta}$ for coefficients $f_{\alpha \beta} \in A(n)^{0}$. Moreover, for $f \in A(n)^{0}$ and $x \in Z$ we have $D_{1,0}(f x)=$ $(d f) x+f D_{1,0} x$. Thus writing $D_{1,0}\left(x_{\alpha}\right)=\sum w_{\alpha \beta} x_{\beta}$ we find

$$
d f_{\alpha \beta}+\sum f_{\alpha \gamma} w_{\gamma \beta}=0, \text { all } \alpha, \beta .
$$

It follows that $d\left(\operatorname{det}\left(f_{\alpha \beta}\right)\right)=\operatorname{det}\left(f_{\alpha \beta}\right) w$, where $w=-\sum_{\beta} w_{\beta \beta}$. The fifth property of admissible simplicial CDGA's now asserts that $\operatorname{det}\left(f_{\alpha \beta}\right)$ is invertible or zero.

But it follows from 3.10 that $\left(f_{\alpha \beta}\right)$ restricts to the identity matrix on $v$. Hence $\operatorname{det}\left(f_{\alpha \beta}\right)$ is invertible and so $\left(f_{\alpha \beta}\right)$ is an invertible matrix; i.e., $\psi_{0}$ is an isomorphism in each degree.

Proof of Theorems 3.7 and 3.8. Consider first Theorem 3.7 We may write $\mathcal{E}(\Phi)=$ $i d \otimes_{R} \varphi: A \otimes_{R}\left(\Lambda Y^{\prime}, D_{0}^{\prime}\right) \rightarrow A \otimes_{R}\left(\Lambda Y, D_{0}\right)$. It follows from the second condition of Definition 3.2 that $A$ is locally free as an $R$-module (Definition 1.9$)$. Hence $\mathcal{E}(\Phi)$ is an isomorphism if and only if $\varphi$ is, and that $\mathcal{E}(\Phi)$ is a quasi-isomorphism if and only if $\varphi$ is. This shows that if either $\varphi$ or $\mathcal{E}(\Phi)$ is a quasi-isomorphism, then $\varphi$, $\mathcal{E}(\Phi)$ and $\Phi$ are isomorphisms.

Next, we establish Theorem 3.8. Here, we may, without loss of generality, suppose that $\mathbf{K}=\boldsymbol{\Delta}^{n}$, and $\sigma=i d$. Let $(\Lambda Z, d)$ be a representative Sullivan algebra for $\left(\Lambda Y, D_{0}\right)$, so that $\left(\Lambda Y, D_{0}\right)=\left(R \otimes_{\mathbf{k}} \Lambda Z, i d \otimes d\right)$. Then restriction to a vertex $v$ is a surjective quasi-isomorphism $\left(A \otimes_{R} \Lambda Y, D\right)_{\sigma} \stackrel{\cong}{\rightarrow}(\Lambda Z, d)$. A CDGA section $(\Lambda Z, d) \rightarrow\left(A \otimes_{R} \Lambda Y, D\right)_{\sigma}$ [21] Corollary 3.6] induces an $A$-quasi-isomorphism $\Phi: A \otimes_{\mathbf{k}}(\Lambda Z, d) \rightarrow\left(A \otimes_{R} \Lambda Y, D\right)$ of $A$-algebras which, by Lemma 3.9, induces an isomorphism $\varphi$. Hence $\Phi$ itself is an isomorphism and Theorem 3.8 is proved. 
We return to the proof of Theorem 3.7. We must show that a quasi-isomorphism $\Phi$ between 1-connected $A$ minimal models of finite type induces an isomorphism $\varphi$. Again it is sufficient to consider the case $\mathbf{K}=\boldsymbol{\Delta}^{n}$. But now Theorem 3.8 allows us to suppose that our minimal models have the form $A \otimes_{\mathbf{k}}(\Lambda Z, d)$, and the result follows from Lemma 3.9.

In view of the local triviality of $\left(A \otimes_{R} \Lambda Y, D\right)$ (Theorem 3.8), the proof of Proposition 2.6](i) gives

Theorem 3.11. Let

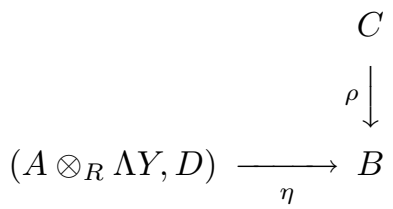

be a diagram of $A$-morphisms. Suppose that $\left(A \otimes_{R} \Lambda Y, D\right)$ is a 1-connected $A$ minimal model of finite type and $\rho$ is a strongly surjective quasi-isomorphism. Then there is an $A$-morphism $\varphi:\left(A \otimes_{R} \Lambda Y, D\right) \rightarrow C$, such that $\rho \varphi=\eta$.

We can now establish existence and uniqueness of minimal models.

Theorem 3.12. Let $j_{E}: A \rightarrow E$ be a 1-connected A-algebra of finite type. Then there are a 1-connected $A$ minimal model $\left(A \otimes_{R} \Lambda Y, D\right)$ of finite type and an $A$ quasi-isomorphism, $m:\left(A \otimes_{R} \Lambda Y, D\right) \stackrel{\simeq}{\rightarrow}$. This condition determines the minimal model uniquely up to A-isomorphism.

Definition 3.13. The morphism $m:\left(A \otimes_{R} \Lambda Y, D\right) \stackrel{\simeq}{\rightrightarrows} E$ (or sometimes just the $A$ minimal model) is called the $A$ minimal model of $E$.

The first step in proving Theorem 3.12 is the analogue of the existence of linear connections in a vector bundle.

Lemma 3.14. Let $Z$ be a locally free $R$-module. Then there is a $\mathbf{k}$-linear map of local systems, $\nabla: Z \rightarrow A^{1} \otimes Z$, such that, for $\sigma \in \mathbf{K}$,

$$
\nabla_{\sigma}(r z)=d r \otimes z+r \nabla_{\sigma} z, r \in R_{\sigma}, \quad z \in Z_{\sigma} .
$$

Proof. We suppose $\nabla$ constructed for all simplices $\tau$ of dimension less than $n$, compatible with the face operators, and the degeneracy operators where defined. Let $\sigma \in \mathbf{K}_{n}$. If $\sigma=s_{i} \tau$, then $Z_{\tau}=R_{\tau} \otimes_{\mathbf{k}} Z$ and $Z_{\sigma}=R_{\sigma} \otimes_{R_{\tau}} Z_{\tau}=R_{\sigma} \otimes_{\mathbf{k}} Z$. Define $\nabla_{\sigma}$ in this case by $\nabla_{\sigma}\left(\sum r_{j} \otimes x_{j}\right)=\sum d r_{j} \otimes x_{j}+\sum r_{j} s_{i}\left(\nabla_{\tau} x_{j}\right)$.

Suppose $\sigma$ is non-degenerate. The construction of $\nabla$ over the $(n-1)$ simplices gives a map $\Gamma(\nabla): Z_{\partial \sigma} \rightarrow\left(A^{1} \otimes_{R} Z\right)_{\partial \sigma}$. Again, by pulling back to $\boldsymbol{\Delta}^{n}$ via $\sigma$ and writing $Z^{\sigma}=R^{\sigma} \otimes_{\mathbf{k}} Z$, we can identify this as a map $\gamma: R_{\partial \sigma} \otimes_{\mathbf{k}} Z \rightarrow$ $\left(A^{1}\right)_{\partial \sigma} \otimes_{\mathbf{k}} Z$. Choose a basis $x_{i}$ of $Z$ and write $\gamma\left(x_{i}\right)=\sum_{j} \gamma_{i j} \otimes x_{j}, \gamma_{i j} \in\left(A^{1}\right)_{\partial \sigma}$. The extendability of $A^{1}$ implies that the $\gamma_{i j}$ extend to elements $\Gamma_{i j}$ in $A(n)^{1}$. Set

$$
\nabla_{\sigma}\left(\sum r_{i} \otimes x_{i}\right)=\sum_{i} d r_{i} \otimes x_{i}+\sum_{i, j} r_{i} \Gamma_{i j} \otimes x_{j}
$$

Definition 3.15. The map $\nabla$ of Lemma 3.14 is called a linear connection in $Z$. 
Proof of Theorem 3.12. We first note that multiplication by $A$ determines an $A$ quasi-isomorphism, $A \otimes_{\mathbf{k}} E \stackrel{\simeq}{\rightrightarrows} E$, which identifies $A \otimes_{\mathbf{k}} E$ as an $A$-algebra under left multiplication by $A$. Hence for existence it will be sufficient to construct a minimal model for $A \otimes_{\mathbf{k}} E$.

Apply Theorem 2.3 to obtain an $R$ minimal model $\varphi:\left(\Lambda Y, D_{0}\right) \stackrel{\simeq}{\rightarrow}\left(R \otimes_{\mathbf{k}} E, d_{E}\right)$. Apply $A \otimes_{R}$ - and identify $A \otimes_{R}\left(R \otimes_{\mathbf{k}} E\right)=A \otimes_{\mathbf{k}} E$ to obtain a morphism of $R$-CDGA's, $\psi_{0}=A \otimes \varphi:\left(A \otimes_{R} \Lambda Y, D_{0}\right) \rightarrow\left(A \otimes_{\mathbf{k}} E, A \otimes d_{E}\right)$. Since each $A(n)$ is a free $A^{0}(n)$-module, $\psi_{0}$ is also a quasi-isomorphism.

Now let $d_{A}$ be the differential in $A$ so that the differential in the $A$-algebra, $A \otimes_{\mathbf{k}} E$, is just $d_{A} \otimes E+A \otimes d_{E}$. We shall perturb $D_{0}$ and $\psi_{0}$ by adding terms involving $A^{i}, i \geq 1$, in order to construct our minimal model. More precisely, choose a linear connection $\nabla$ in $Y$. Extend $\nabla+D_{0}: Y \rightarrow A \otimes_{R} \Lambda Y$ (uniquely) to a derivation, $\delta_{0}$, of degree 1 , such that $\delta_{0}$ restricts to $d_{A}$ in $A$. We shall extend $\left(\delta_{0}, \psi_{0}\right)$ to a sequence $\left(\delta_{j}, \psi_{j}\right)_{j \geq 0}$ such that for $j \geq 1$ :

(a) $\delta_{j}$ is a derivation in the local system $A \otimes_{R} \Lambda Y$, and $\delta_{j}-\delta_{j-1}$ vanishes on $A$ and sends $Y$ to $A^{\geq j} \otimes_{R} \Lambda Y$;

(b) $\psi_{j}: A \otimes_{R} \Lambda Y \rightarrow A \otimes_{\mathbf{k}} E$ is a morphism of local systems of graded algebras, and $\psi_{j}-\psi_{j-1}$ vanishes on $A$ and sends $Y$ to $A^{\geq j} \otimes_{\mathbf{k}} E$;

(c) $\delta_{j}^{2}: Y \rightarrow A^{\geq j+1} \otimes_{R} \Lambda Y$;

(d) $d \psi_{j}-\psi_{j} \delta_{j}: Y \rightarrow A^{\geq j+1} \otimes_{\mathbf{k}} E$.

First, we note that left and right multiplication make each $\left(A^{i} \otimes_{R} \Lambda Y, D_{0}\right)$ into a $\left(\Lambda Y, D_{0}\right)$-bimodule (cf. Definition 2.12). Moreover, we may use $\varphi$ to regard $A \otimes_{\mathbf{k}} E$ as an algebra over $\Lambda Y$. Thus left and right multiplication also make each $\left(A^{i} \otimes_{\mathbf{k}} E, d_{E}\right)$ into a $\left(\Lambda Y, D_{0}\right)$-bimodule, and $\psi_{0}:\left(A^{i} \otimes_{R} \Lambda Y, D_{0}\right) \rightarrow\left(A^{i} \otimes_{\mathbf{k}} E, d_{E}\right)$ is a quasi-isomorphism of bimodules.

Next note that the couple $\left(\delta_{0}, \psi_{0}\right)$ does in fact satisfy conditions (a)-(d). Suppose $\left(\delta_{j}, \psi_{j}\right)$ constructed for some $j \geq 0$. Then $\delta_{j}^{2}$ is a derivation which vanishes on $A$, and so it can be uniquely written as the sum of two such derivations, one of which sends $\Lambda Y$ to $A^{j+1} \otimes_{R} \Lambda Y$, and the other of which maps $\Lambda Y \rightarrow A^{\geq j+2} \otimes_{R} \Lambda Y$. Call the first derivation $f$.

Similarly, $d \psi_{j}-\psi_{j} \delta_{j}$ vanishes on $A$ and maps $Y$ into $A^{\geq j+1} \otimes_{\mathbf{k}} E$. Thus its restriction to $Y$ is sum of two $R$ linear maps, the first sending $Y$ into $A^{j+1} \otimes_{\mathbf{k}} E$ and the second sending $Y$ into $A^{\geq j+2} \otimes_{\mathbf{k}} E$. Extend the first map to a derivation $g \in \operatorname{Der}\left(\Lambda Y, A^{j+1} \otimes_{\mathbf{k}} E\right)$.

A straightforward calculation now shows that $D_{0} f-f D_{0}=0$ and $\psi_{0} \circ f=$ $d_{E} g+g D_{0}$.

On the other hand, Proposition 2.14, applied with $M=\left(A^{j+1} \otimes_{R} \Lambda Y, D_{0}\right)$ and $N=\left(A^{j+1} \otimes_{\mathbf{k}} E, d_{E}\right)$, asserts that composition with $\psi_{0}$ defines a quasiisomorphism from $\operatorname{Der}_{R}\left(\Lambda Y, A^{j+1} \otimes_{R} \Lambda Y\right)$ to $\operatorname{Der}_{R}\left(\Lambda Y, A^{j+1} \otimes_{\mathbf{k}} E\right)$. Hence we can find derivations $\theta \in \operatorname{Der}_{R}\left(\Lambda Y, A^{j+1} \otimes_{R} \Lambda Y\right), \xi \in \operatorname{Der}_{R}\left(\Lambda Y, A^{j+1} \otimes_{\mathbf{k}} E\right)$, such that $D_{0} \theta+\theta D_{0}=f$ and $\psi_{0} \circ \theta=g+d_{E} \xi-\xi D_{0}$.

Now define $\delta_{j+1}$ and $\psi_{j+1}$ by the conditions $\delta_{j+1_{\mid Y}}=\delta_{j}-\theta$ and $\psi_{j+1}{ }_{\mid Y}=\psi_{j}-\xi$. An easy verification shows that (c) and (d) are satisfied.

Finally, set $D=\lim _{j} \delta_{j}$ and $m=\lim _{j} \psi_{j}$. Formulae (a) and (b) identify $\mathcal{E}(m)$ with the quasi-isomorphism $\psi_{0}$. Thus $m$ is a quasi-isomorphism by Theorem 3.7 .

Given the Lifting Theorem 3.11, and the Isomorphism Theorem 3.7, the proof of uniqueness is identical with the proof in Theorem 2.3 . 
Let $m:\left(A \otimes_{R} \Lambda Y, D\right) \rightarrow E$ be an $A$ minimal model for an $A$-algebra $E$, and write $D=D_{0}+D_{1}+\cdots$ as above. Thus $D_{1}: \Lambda Y \rightarrow A^{1} \otimes_{R} \Lambda Y$ is a linear connection in the $R$-module $\Lambda Y$, satisfying $D_{1} D_{0}+D_{0} D_{1}=0$.

Definition 3.16. $\left(\Lambda Y, D_{0}\right)$ is the fibre of the minimal model and $D_{1}$ is a distinguished connection corresponding to the $A$-algebra $E$.

From the uniqueness of minimal models we deduce

Proposition 3.17. The set of distinguished connections in $\left(\Lambda Y, D_{0}\right)$ corresponding to $E$ consists of the operators $D_{1}^{\prime}$ satisfying $(\mathrm{id} \otimes \varphi) D_{1}^{\prime}(\mathrm{id} \otimes \varphi)^{-1}-D_{1}=D_{0} h-h D_{0}$, where $h: \Lambda Y \rightarrow A^{1} \otimes_{R} \Lambda Y$ is a $\Lambda Y$-derivation and $\varphi$ is an automorphism of $\left(\Lambda Y, D_{0}\right)$.

\section{4. Номотору}

In this section, we work over a field $\mathbf{k}$ of characteristic zero and with the category of connected simplicial sets, $\mathbf{K} . A(*)$ is an admissible simplicial CDGA with coefficients in $\mathbf{k}$ and $R(*)$ is the simplicial algebra $A(*)^{0}$.

Consider the CDGA's $\Lambda(t, d t)$ and $\Lambda(t, s, d t, d s)$ where $t$ and $s$ are variables of degree zero. They determine constant local systems $I$ and $T$ on $\mathbf{K}, I_{\sigma}=\Lambda(t, d t)$, $T_{\sigma}=\Lambda(t, s, d t, d s), \sigma \in \mathbf{K}$, with all face and degeneracy operators reducing to the identity. Define $\varepsilon_{0}, \varepsilon_{1}: I \rightarrow \mathbf{k}$ and $\partial_{0}, \partial_{1}, \partial_{2}: T \rightarrow I$, by $\varepsilon_{0}(t)=0, \varepsilon_{1}(t)=1$; $\partial_{0}(t)=1, \partial_{0}(s)=t ; \partial_{1}(t)=t, \partial_{1}(s)=t ; \partial_{2}(t)=t, \partial_{2}(s)=0$.

Definition 4.1. (1) The cylinder for an $A$-algebra, $E$, is the $A$-algebra $E \otimes_{\mathbf{k}} I$, together with the retractions $\varepsilon_{i}=E \otimes_{\mathbf{k}} \varepsilon_{i}: E \otimes_{\mathbf{k}} I \rightarrow E$.

(2) Let $\varphi_{0}, \varphi_{1}:\left(A \otimes_{R} \Lambda Y, D\right) \rightarrow E$ be $A$-morphisms (over $\mathbf{K}$ ), with source a 1-connected $A$ minimal model of finite type. Then $\varphi_{0}$ and $\varphi_{1}$ are homotopic over $\mathbf{K}$ (we write $\left.\varphi_{0} \sim_{\mathbf{K}} \varphi_{1}\right)$ if there is an $A$-morphism $\Phi:\left(A \otimes_{R} \Lambda Y, D\right) \rightarrow E \otimes_{\mathbf{K}} I$ such that $\varepsilon_{i} \Phi=\varphi_{i}, i=0,1$. The morphism $\Phi$ is called an $A$-homotopy from $\varphi_{0}$ to $\varphi_{1}$.

Lemma 4.2. (i) Suppose given a sequence of A-morphisms $\left(A \otimes_{R} \Lambda Y^{\prime}, D^{\prime}\right) \stackrel{g}{\longrightarrow}$ $\left(A \otimes_{R} \Lambda Y, D\right) \stackrel{\stackrel{\varphi_{0}}{\varphi_{1}}}{\rightrightarrows} E \stackrel{h}{\longrightarrow} E^{\prime}$, with the first two objects 1-connected $A$ minimal models of finite type. Then $\varphi_{0} \sim_{\mathbf{K}} \varphi_{1} \Rightarrow h \varphi_{0} g \sim_{\mathbf{K}} h \varphi_{1} g$.

(ii) A-homotopy is an equivalence relation.

Proof. (i) is obvious, as is the reflexivity of $A$-homotopy. Symmetry follows from the fact that the isomorphism $t \mapsto 1-t, d t \mapsto-d t$ of $I$ interchanges $\varepsilon_{0}$ and $\varepsilon_{1}$. For transitivity, suppose given $\varphi_{0}, \varphi_{1}, \varphi_{2}:\left(A \otimes_{R} \Lambda Y, D\right) \rightarrow E$ with $\varphi_{0} \sim_{\mathbf{K}} \varphi_{1}$ and $\varphi_{1} \sim_{\mathbf{K}} \varphi_{2}$ via homotopies $\Phi_{1}$ and $\Phi_{2}$. Let $I^{\prime}$ be a second copy of $I$ and consider the fibre product $I \times_{\mathbf{k}} I^{\prime}$ with respect to $\varepsilon_{1}: I \rightarrow \mathbf{k}$ and $\varepsilon_{0}: I^{\prime} \rightarrow \mathbf{k}$. Since tensor products commute with fibre products, $\Phi_{1}$ and $\Phi_{2}$ define an $A$-morphism $\left(\Phi_{1}, \Phi_{2}\right)$ : $\left(A \otimes_{R} \Lambda Y, D\right) \rightarrow E \otimes_{\mathbf{k}}\left(I \times_{\mathbf{k}} I^{\prime}\right)$. Put $\rho=\left(\partial_{2}, \partial_{0}\right): T \rightarrow I \times_{\mathbf{k}} I^{\prime}$. A trivial calculation shows that $\rho$ is surjective, and clearly ker $\rho$ is again a constant local system. Then $E \otimes_{\mathbf{k}} \rho: E \otimes_{\mathbf{k}} T \rightarrow E \otimes_{\mathbf{k}}\left(I \times_{\mathbf{k}} I^{\prime}\right)$ is strongly surjective because $E$ is extendable and $k e r \rho$ is constant (Proposition 1.14). Thus by Theorem 3.11, $\left(\Phi_{1}, \Phi_{2}\right)$ lifts through the quasi-isomorphism $E \otimes_{\mathbf{k}} \rho$ to an $A$-morphism $\Omega:\left(A \otimes_{R} \Lambda Y, D\right) \rightarrow E \otimes_{\mathbf{k}} T$. Now $\left(E \otimes_{\mathbf{k}} \partial_{1}\right) \circ \Omega$ is the desired $A$-homotopy from $\varphi_{0}$ to $\varphi_{2}$.

Let $\left(A \otimes_{R} \Lambda Y, D\right)$ and $E$ be respectively a 1-connected $A$ minimal model of finite type and an $A$-algebra. We will denote by $\left[\left(A \otimes_{R} \Lambda Y, D\right), E\right]$ the set of $A$-homotopy 
classes of $A$-morphisms. Lemma 4.2 shows that if $E^{\prime}$ is a second $A$-algebra, then an $A$-morphism $\varphi: E^{\prime} \rightarrow E$ induces a set map

$$
\varphi_{\sharp}:\left[\left(A \otimes_{R} \Lambda Y, D\right), E^{\prime}\right] \rightarrow\left[\left(A \otimes_{R} \Lambda Y, D\right), E\right] .
$$

Theorem 4.3. If $\varphi$ is a quasi-isomorphism, then $\varphi_{\sharp}$ is a bijection.

Proof. First consider the case that $\varphi$ is strongly surjective. It follows immediately from the Lifting Theorem[3.11 that $\varphi_{\sharp}$ is surjective. Observe now that $A$-morphisms $f_{0}, f_{1}:\left(A \otimes_{R} \Lambda Y, D\right) \rightarrow E$, together with an $A$-homotopy $\Phi$ from $\varphi f_{0}$ to $\varphi f_{1}$, define an $A$-morphism $\left(\Phi ; f_{0}, f_{1}\right):\left(A \otimes_{R} \Lambda Y, D\right) \rightarrow\left(E \otimes_{\mathbf{k}} I\right) \times_{E \times E}\left(E^{\prime} \times E^{\prime}\right)$. Moreover the kernel of the $A$-morphism $E^{\prime} \otimes_{\mathbf{k}} I \stackrel{\left(\varphi \otimes i d ; \varepsilon_{0}, \varepsilon_{1}\right)}{\longrightarrow}\left(E \otimes_{\mathbf{k}} I\right) \times_{E \times E}\left(E^{\prime} \times E^{\prime}\right)$ is $\operatorname{ker} \varphi \otimes_{\mathbf{k}} \operatorname{ker}\left(\varepsilon_{0}, \varepsilon_{1}\right)$. Since $\varphi$ is a strongly surjective quasi-isomorphism and $E^{\prime}$ is extendable, $\operatorname{ker} \varphi$ is extendable (Proposition 1.14) and $H(\operatorname{ker} \varphi)=0$. Since $\operatorname{ker}\left(\varepsilon_{0}, \varepsilon_{1}\right)$ is a constant system, these properties extend to $\operatorname{ker} \varphi \otimes_{\mathbf{k}} \operatorname{ker}\left(\varepsilon_{0}, \varepsilon_{1}\right)$. Thus the morphism $\left(\varphi \otimes_{\mathbf{k}} i d ; \varepsilon_{0}, \varepsilon_{1}\right)$ is also a strongly surjective quasi-isomorphism. Lift $\left(\Phi ; f_{0}, f_{1}\right)$ through this morphism (Theorem 3.11) to obtain an $A$-homotopy from $f_{0}$ to $f_{1}$.

Consider the general case. Define $(\mathcal{U}(E), \delta)$ by $\mathcal{U}(E)_{\sigma}=\Lambda\left(E_{\sigma} \oplus V_{\sigma}\right), \delta: E_{\sigma} \cong$ $V_{\sigma}$, and a morphism $\pi:(\mathcal{U}(E), \delta) \rightarrow E$ by $\pi_{\mid E}=$ identity. Now factor $\varphi$ as the composite of the $A$-morphisms $E^{\prime} \stackrel{\lambda}{\rightarrow} E^{\prime} \otimes_{\mathbf{k}} \mathcal{U}(E) \stackrel{\varphi \cdot \pi}{\longrightarrow} E$. The map $\lambda$ is a quasiisomorphism and $\varphi . \pi$ is a strongly surjective quasi-isomorphism. Thus by the first part of the proof, $(\varphi \cdot \pi)_{\sharp}$ is a bijection. It remains to show that $\lambda_{\sharp}$ is a bijection. Define $\rho: E^{\prime} \otimes_{\mathbf{k}} \mathcal{U}(E) \rightarrow E^{\prime}$ by setting $\rho=0$ in $E$ and in $V$. Then $\rho$ is a strongly surjective quasi-isomorphism and so $\rho_{\sharp}$ is a bijection. Since $\rho \lambda=i d, \lambda_{\sharp}$ is the inverse.

Definition and Remark 4.4. (1) Let $f_{0}, f_{1}: E \rightarrow E^{\prime}$ be $A$-morphisms with $E$

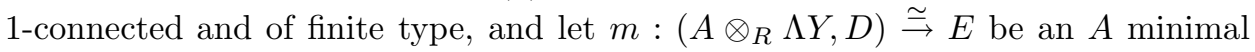
model. We say $f_{0}$ and $f_{1}$ are $A$-homotopic and write $f_{0} \sim_{\mathbf{K}} f_{1}$ if $f_{0} \circ m \sim_{\mathbf{K}} f_{1} \circ m$.

(2) It follows immediately from Theorem 4.3 that $A$-homotopy is independent of the choice of $m$, and Lemma 4.2 (ii) shows that it is an equivalence relation. The set of homotopy classes of $A$-morphisms from $E$ to $E^{\prime}$, with $E$ simply connected and of finite type, will be denoted by $\left[E, E^{\prime}\right]$.

Finally Lemma 4.2 (i) and Theorem 4.3 extend immediately to the

Proposition 4.5. Let $g: E \rightarrow E^{\prime}$ and $h: E^{\prime \prime} \rightarrow E^{\prime \prime \prime}$ be A-morphisms, with $E, E^{\prime}$ simply connected and of finite type.

(i) Precomposition with $g$ and composition with $h$ define maps $g^{\sharp}:[E,-] \leftarrow$ $\left[E^{\prime},-\right]$ and $h_{\sharp}:\left[-, E^{\prime \prime}\right] \rightarrow\left[-, E^{\prime \prime \prime}\right]$.

(ii) If $g$ and $h$ are quasi-isomorphisms, then $g^{\sharp}$ and $h_{\sharp}$ are injections.

We end this section by constructing an action 'up to homotopy' of the fundamental group of $\mathbf{K}$ on the fibre of an $A$ minimal model. Let $\left(A \otimes_{R} \Lambda Y, D\right)$ be a 1-connected $A$ minimal model of finite type. For any edge $\tau \in \mathbf{K}_{1}$, from $v_{0}$ to $v_{1}$, we have the quasi-isomorphisms:

$$
(\Lambda Z, d)_{v_{0}} \stackrel{\stackrel{\partial_{1}}{\simeq}}{\simeq}\left(A \otimes_{R} \Lambda Y, D\right)_{\tau} \stackrel{\partial_{0}}{\simeq}(\Lambda Z, d)_{v_{1}} .
$$

By the classical lifting property, there exists $\alpha_{\tau}:(\Lambda Z, d)_{v_{0}} \rightarrow\left(A \otimes_{R} \Lambda Y, D\right)_{\tau}$ such that $\partial_{1} \circ \alpha_{\tau} \sim_{\mathbf{K}} i d$ and the homotopy class $\left[\alpha_{\tau}\right]$ is uniquely defined (4.3). This gives 
a quasi-isomorphism $\partial_{0} \circ \alpha_{\tau}:(\Lambda Z, d)_{v_{0}} \rightarrow(\Lambda Z, d)_{v_{1}}$ (therefore an isomorphism) the homotopy class of which is well defined. Consider the monoid

$$
\Omega \mathbf{K}_{v}=\left\{\left(\sigma_{0}, \sigma_{1}, \ldots, \sigma_{n}\right) \mid \sigma_{n}(0)=\sigma_{0}(1) ; \sigma_{i}(0)=\sigma_{i-1}(1), 0 \leq i \leq n-1\right\}
$$

with the usual composition. Denote by $\pi_{0} \operatorname{Aut}(\Lambda Z, d)_{v}$ the group of homotopy classes of automorphisms of $(\Lambda Z, d)_{v}$. This construction defines a homomorphism $p: \Omega \mathbf{K}_{v} \rightarrow \pi_{0} \operatorname{Aut}(\Lambda Z, d)_{v}$. Moreover, if $\Delta$ is a 2-simplex in $\mathbf{K}$ with edges $\partial_{2} \Delta=\sigma_{i}$, $\partial_{0} \Delta=\sigma_{i+1}$ and $\partial_{1} \Delta=\tau$, then

$$
p\left(\sigma_{0}, \ldots, \sigma_{i}, \sigma_{i+1}, \ldots, \sigma_{n}\right)=p\left(\sigma_{0}, \ldots, \sigma_{i-1}, \tau, \sigma_{i+2}, \ldots, \sigma_{n}\right) .
$$

Thus $p$ induces a representation of $\pi_{1}(\mathbf{K}, v)$ [16] [Chapter $1, \S 3$ ]:

$$
\rho: \pi_{1}(\mathbf{K}, v) \rightarrow \pi_{0} \operatorname{Aut}(\Lambda Z, d) .
$$

As a consequence of (4.6), one has structures of $\pi_{1}(\mathbf{K}, v)$-modules on $H(\Lambda Z, d)$ and $\operatorname{Hom}\left(Z^{k}, \mathbf{k}\right)$ induced by the natural maps $\pi_{0}(A u t(\Lambda Z, d)) \stackrel{H_{A u t}}{\longrightarrow} A u t H(\Lambda Z, d)$ and $\pi_{0}(A u t(\Lambda Z, d)) \stackrel{\pi_{A u t}}{\longrightarrow} A u t \pi_{*}(\Lambda Z, d)$ where $\pi_{*}(\Lambda Z, d)=\Lambda^{+} Z / \Lambda^{+} Z . \Lambda^{+} Z$.

On the other hand, $\rho$ does not always lift to a representation $\pi_{1}(\mathbf{K}, v) \rightarrow$ $\operatorname{Aut}(\Lambda Z, d)$. In fact even $H_{A u t} \circ \rho$ may not lift, as we show in Example6.7.

\section{KAN FIBRATIONS}

We continue the conventions of §马: We work over a fixed field $\mathbf{k}$ of characteristic zero and with the category of connected simplicial sets $\mathbf{K} . A(*)$ is an admissible simplicial CDGA with coefficients in $\mathbf{k}, R(*)$ the simplicial algebra $A(*)^{0}$ and $A$ the local system $A_{\mathbf{K}}$ on $\mathbf{K}$ determined by $A(*)$. At the end of this section, for Theorems 5.10 and [5.11] we specialize to $\mathbf{k}=\mathbb{Q}$.

For any simplicial set $\mathbf{L}$ we will denote by $A(\mathbf{L})$ the $\operatorname{CDGA} \Gamma\left(A_{\mathbf{L}}\right)$ of global sections of $A_{\mathbf{L}}$.

Proposition 5.1. There are natural isomorphisms of graded algebras $H^{*}(A(\mathbf{L})) \cong$ $H^{*}(\mathbf{L} ; \mathbf{k})$ for all simplicial sets $\mathbf{L}$.

Proof. The morphisms $A_{P L}(*) \rightarrow A_{P L}(*) \otimes A(*) \leftarrow A(*)$ are quasi-isomorphisms of extendable simplicial CDGA's [13, Theorem 12.37]. Thus they define natural isomorphisms $H\left(A_{P L}(\mathbf{L})\right) \cong H(A(\mathbf{L}))$. On the other hand, $H\left(A_{P L}(\mathbf{L})\right)$ is naturally isomorphic with $H^{*}(\mathbf{L} ; \mathbf{k})$ [21 Theorem 7.1].

Definition 5.2. A simplicial set over $\mathbf{K}$ is a simplicial map $\mathbf{E} \stackrel{\mathbf{p}}{\rightarrow} \mathbf{K}$, denoted by $(\mathbf{E}, \mathbf{p})$. A morphism $\mathbf{u}:(\mathbf{E}, \mathbf{p}) \rightarrow\left(\mathbf{E}^{\prime}, \mathbf{p}^{\prime}\right)$, between simplicial sets over $\mathbf{K}$, is a simplicial map, $\mathbf{u}: \mathbf{E} \rightarrow \mathbf{E}^{\prime}$, such that $\mathbf{p}^{\prime} \circ \mathbf{u}=\mathbf{p}$.

Given a simplicial map p : $\mathbf{E} \rightarrow \mathbf{K}$ and $\sigma \in \mathbf{K}_{n}$ we denote the pull-back over $\sigma$ by $\mathbf{p}^{\sigma}: \mathbf{E}^{\sigma} \rightarrow \boldsymbol{\Delta}^{n}$. The restriction to $\partial \boldsymbol{\Delta}^{n}$ is denoted by $\mathbf{p}^{\partial \sigma}: \mathbf{E}^{\partial \sigma} \rightarrow \partial \boldsymbol{\Delta}^{n}$. We say $\mathbf{p}$ is a locally trivial fibre bundle if, for each $\sigma \in \mathbf{K}_{n}$, there are simplicial isomorphisms $\mathbf{E}^{\sigma} \cong \boldsymbol{\Delta}^{n} \times \mathbf{F}(\sigma)$ that convert $\mathbf{p}^{\sigma}$ to the obvious projection. In this case, since $\mathbf{K}$ is connected, each $\mathbf{F}(\sigma)$ is isomorphic with a single simplicial set $\mathbf{F}$, called the typical fibre of $\mathbf{p}$. A locally trivial bundle is a Kan fibration if and only if the typical fibre is a Kan complex.

We establish the following notation: $\mathcal{S}_{\mathbf{K}}$ is the category of simplicial sets over $\mathbf{K}$; $\mathcal{K}_{\mathbf{K}}$ is the full subcategory of Kan fibrations with simply connected fibres; $\mathcal{K}_{\mathbf{K}}^{f}$ is the full subcategory of $\mathcal{K}_{\mathbf{K}}$ in which the rational homology of the fibre has finite type. Recall that $\mathcal{A}_{\mathbf{K}}$ (resp. $\mathcal{A}_{\mathbf{K}}^{f}$ ) denotes the category of $A$-algebras (resp. 1-connected 
$A$-algebras of finite type) on $\mathbf{K}$ and $A$-morphisms, and that $\mathcal{M}_{\mathbf{K}}$ denotes the full subcategory of 1-connected $A$ minimal models of finite type.

We now define contravariant functors $\mathcal{F}: \mathcal{K}_{\mathbf{K}} \rightarrow \mathcal{A}_{\mathbf{K}}$ and \langle\rangle$: \mathcal{A}_{\mathbf{K}} \rightarrow \mathcal{S}_{\mathbf{K}}$ satisfying an adjointness condition compatible with the relations of homotopy. When $\mathbf{K}=\mathbf{p t}$, there are precisely Sullivan's constructions [21, §8] and for arbitrary $\mathbf{K}$ both the constructions and the verifications of their properties are the obvious generalizations.

To construct $\mathcal{F}$ let $\mathbf{p}: \mathbf{E} \rightarrow \mathbf{K}$ be any simplicial set over $\mathbf{K}$. If $\sigma \in \mathbf{K}$, there are canonical simplicial maps $\gamma_{i}: \mathbf{E}^{\partial_{i} \sigma} \rightarrow \mathbf{E}^{\sigma}$ and $\boldsymbol{\omega}_{j}: \mathbf{E}^{s_{j} \sigma} \rightarrow \mathbf{E}^{\sigma}$, satisfying the usual commutation relations, and covering the standard face and degeneracy maps $\boldsymbol{\partial}_{i}: \boldsymbol{\Delta}^{|\sigma|-1} \rightarrow \boldsymbol{\Delta}^{|\sigma|}$ and $\mathbf{s}_{j}: \boldsymbol{\Delta}^{|\sigma|+1} \rightarrow \boldsymbol{\Delta}^{|\sigma|}$. Thus a local system $\mathcal{F}(\mathbf{E}, \mathbf{p})$ of CDGA's on $\mathbf{K}$ is defined by $\mathcal{F}(\mathbf{E}, \mathbf{p})_{\sigma}=A\left(\mathbf{E}^{\sigma}\right)$ and with face and degeneracy operators $A\left(\boldsymbol{\gamma}_{i}\right)$ and $A\left(\boldsymbol{\omega}_{j}\right)$. Moreover, a morphism $j: A \rightarrow \mathcal{F}(\mathbf{E}, \mathbf{p})$ is given by $j_{\sigma}=A\left(\mathbf{p}^{\sigma}\right): A(|\sigma|)=A\left(\boldsymbol{\Delta}^{|\sigma|}\right) \rightarrow A\left(\mathbf{E}^{\sigma}\right)$. Note that if $\mathbf{E}=\mathbf{K}$, then $\mathcal{F}(\mathbf{E}, \mathbf{p})=A$ and that if $\mathbf{K}=\mathbf{p t}$, then $\mathcal{F}(\mathbf{E}, \mathbf{p})$ is the CDGA, $A(\mathbf{E})$. It is immediate from the construction that $\mathcal{F}$ commutes with pull-backs.

Proposition 5.3. Let $\mathbf{p}: \mathbf{E} \rightarrow \mathbf{K}$ be a Kan fibration. Then

(i) $\Gamma(\mathcal{F}(\mathbf{E}, \mathbf{p}))=A(\mathbf{E})$.

(ii) $(\mathcal{F}(\mathbf{E}, \mathbf{p}), j)$ is an A-algebra on $\mathbf{K}$. If the Kan fibration is in $\mathcal{K}_{\mathbf{K}}^{f}$, then the A-algebra is in $\mathcal{A}_{\mathbf{K}}^{f}$.

Proof. (i) Recall that a simplex in $\mathbf{E}^{\sigma}$ is a pair $(\omega, \tau)$ where $\omega$ is a simplex in $\boldsymbol{\Delta}^{|\sigma|}$, $\tau \in \mathbf{E}$ and $\boldsymbol{\sigma}(\omega)=\mathbf{p}(\tau)$. Now a global section $f \in \Gamma(\mathcal{F}(\mathbf{E}, \mathbf{p}))$ is a function that assigns to each $\sigma \in \mathbf{K}$ an element $f_{\sigma} \in A\left(\mathbf{E}^{\sigma}\right)$, compatible with $A\left(\gamma_{i}\right)$ and $A\left(\boldsymbol{\omega}_{j}\right)$. The corresponding element $g \in A(\mathbf{E})$ is given by $g_{\tau}=\left(f_{\mathbf{p}(\tau)}\right)_{(\mathbf{p}(\tau), \tau)}$. It is obvious (cf. 13, Lemma 19.21] for the case $A=A_{P L}$ ) that this defines an isomorphism from $\Gamma(\mathcal{F}(\mathbf{E}, \mathbf{p}))$ to $A(\mathbf{E})$.

(ii) Since $\mathbf{p}$ is a Kan fibration, the $\boldsymbol{\gamma}_{i}$ and $\boldsymbol{\omega}_{j}$ induce isomorphisms of homotopy and homology groups. Hence by Proposition $5.1 A\left(\boldsymbol{\gamma}_{i}\right)$ and $A\left(\boldsymbol{\omega}_{j}\right)$ are quasiisomorphisms and so $H(\mathcal{F}(\mathbf{E}, \mathbf{p}))$ is locally constant. Since $A$ is extendable, the restrictions $A\left(\mathbf{E}^{\sigma}\right) \rightarrow A\left(\mathbf{E}^{\partial \sigma}\right)$ are surjective. By (i) these are identified with the restrictions $\mathcal{F}(\mathbf{E}, \mathbf{p})_{\sigma} \rightarrow \mathcal{F}(\mathbf{E}, \mathbf{p})_{\partial \sigma}$ and so $\mathcal{F}(\mathbf{E}, \mathbf{p})$ is extendable. If the Kan fibration is in $\mathcal{K}_{\mathbf{K}}^{f}$, then the fibre $\mathbf{E}^{\mathbf{v}}$ at a vertex $\mathbf{v}$ is simply connected and has homology of finite type. Thus $H\left(A\left(\mathbf{E}^{\mathbf{v}}\right)\right)$ also has these properties, and so $\mathcal{F}(\mathbf{E}, \mathbf{p})$ is simply connected of finite type.

To construct the realisation functor \langle\rangle let $j_{E}: A \rightarrow E$ be an $A$-algebra on $\mathbf{K}$, define the simplices of $\left\langle E, j_{E}\right\rangle$ by $\left\langle E, j_{E}\right\rangle_{n}=\left\{(\varphi, \sigma) \mid \sigma \in \mathbf{K}_{n} ; \varphi \in \mathcal{A}_{\Delta^{n}}\left(E^{\sigma}, A_{\Delta^{n}}\right)\right\}$. The face and degeneracy operators are defined by $\partial_{i}(\varphi, \sigma)=\left(\partial_{i} \varphi, \partial_{i} \sigma\right)$ and $s_{j}(\varphi, \sigma)$ $=\left(s_{j} \varphi, s_{j} \sigma\right)$, where $\partial_{i} \varphi$ and $s_{j} \varphi$ are the respective pull-backs of $\varphi$ via the simplicial maps $\boldsymbol{\partial}_{i}: \boldsymbol{\Delta}^{n-1} \rightarrow \boldsymbol{\Delta}^{n}$ and $\mathbf{s}_{j}: \boldsymbol{\Delta}^{n+1} \rightarrow \boldsymbol{\Delta}^{n}$. The projection $\langle E, j\rangle \stackrel{\mathbf{p}}{\rightarrow} \mathbf{K}$ is given by $\mathbf{p}(\varphi, \sigma)=\sigma$. Again note that \langle\rangle commutes with pull-backs. When $\mathbf{K}=\mathbf{p t}$, then an $A$-algebra is just a CDGA, $C$, and its realization $\langle C\rangle$ is precisely as defined in $[21, \S 8]$ both in the case $A(*)=A_{P L}(*)$ and $A(*)=A_{D R}(*)$.

Theorem 5.4. For Kan fibrations $\mathbf{p}: \mathbf{E} \rightarrow \mathbf{K}$ and $A$-algebras $j_{E}: A \rightarrow E$ there is a natural bijection: $S_{\mathbf{K}}\left((\mathbf{E}, \mathbf{p}),\left\langle E, j_{E}\right\rangle\right) \cong \mathcal{A}_{\mathbf{K}}\left(\left(E, j_{E}\right), \mathcal{F}(\mathbf{E}, \mathbf{p})\right)$.

Proof. The left-hand side consists of the functions that assign to each $\tau \in \mathbf{E}$ a morphism $f_{\tau} \in \mathcal{A}_{\boldsymbol{\Delta}^{|\tau|}}\left(E^{\mathbf{p}(\tau)}, A_{\boldsymbol{\Delta}}^{|\tau|}\right)$, compatible with the face and degeneracy 
operators. The correspondence $f_{\tau} \leftrightarrow h_{\tau}=\left(f_{\tau}\right)_{\mathbf{p}(\tau)}: E_{\mathbf{p}(\tau)} \rightarrow A(|\tau|)$ identifies this with the set of functions that assign to each $\tau \in \mathbf{E}$ a DGA homomorphism $h_{\tau}: E_{\mathbf{p}(\tau)} \rightarrow A(|\tau|)$, inducing the identity in $A(|\tau|)$, and such that $h_{\partial_{i} \tau} \circ \partial_{i}=\partial_{i} \circ h_{\tau}$ and $h_{s_{j} \tau} \circ s_{j}=s_{j} \circ h_{\tau}$.

On the other hand, the right side consists of functions that assign to each $\sigma \in \mathbf{K}$ a DGA homomorphism $g_{\sigma}: E_{\sigma} \rightarrow A\left(\mathbf{E}^{\sigma}\right)$, compatible with the face and degeneracy operators, and inducing the identity in $A(|\sigma|)$. Now a simplex in $\mathbf{E}^{\sigma}$ is a pair $(\omega, \tau)$ where $\omega$ is a simplex in $\boldsymbol{\Delta}^{|\sigma|}, \tau \in \mathbf{E}$ and $\boldsymbol{\sigma}(\omega)=\mathbf{p}(\tau)$. Moreover $\omega$ induces a DGA morphism $E_{\sigma} \stackrel{\omega}{\longrightarrow} E_{\boldsymbol{\sigma}(\omega)}$. Thus given a family $\left(h_{\tau}\right)_{\tau \in \mathbf{E}}$ as above we can define a corresponding family $g_{\sigma}$ by $\left(g_{\sigma} \Phi\right)_{(\omega, \tau)}=h_{\tau}(\omega(\Phi))$. This is the desired bijection.

Proposition 5.5. Let $\left(A \otimes_{R} \Lambda Y, D\right)$ be a 1-connected $A$ minimal model of finite type with representative minimal Sullivan algebra $(\Lambda Z, d)$. Then $\left\langle\left(A \otimes_{R} \Lambda Y, D\right), j\right\rangle$ $\rightarrow \mathbf{K}$ is a locally trivial fibre bundle with fibre the Kan complex $\langle\Lambda Z, d\rangle$. In particular, it is an object of $\mathcal{K}_{\mathbf{K}}^{f}$.

Proof. It suffices to consider the case when $\mathbf{K}=\boldsymbol{\Delta}^{n}$. In this case, by Theorem 3.8 we may write $\left(A \otimes_{R} \Lambda Y, D\right)=A \otimes_{\mathbf{k}}(\Lambda Z, d)$, whence $\left\langle\left(A \otimes_{R} \Lambda Y, D\right), j\right\rangle=\boldsymbol{\Delta}^{n} \times$ $\langle\Lambda Z, d\rangle$. We have only to verify that $\langle\Lambda Z, d\rangle$ is a Kan complex, since it is clearly 1-connected. A simplicial map $\phi: \boldsymbol{\Delta}^{n, k} \rightarrow\langle\Lambda Z, d\rangle$ corresponds by adjunction to a CDGA morphism $\psi:(\Lambda Z, d) \rightarrow A\left(\boldsymbol{\Delta}^{n, k}\right)$. Inclusion induces a surjective quasi-isomorphism $\mu: A\left(\boldsymbol{\Delta}^{n}\right) \rightarrow A\left(\boldsymbol{\Delta}^{n, k}\right)$. Thus $\psi$ lifts through $\mu$ to a morphism $\bar{\psi}:(\Lambda Z, d) \rightarrow A\left(\boldsymbol{\Delta}^{n}\right)$. By adjunction, we get the extension of $\psi$.

Let $\boldsymbol{\lambda}_{0}, \boldsymbol{\lambda}_{1}: \boldsymbol{\Delta}^{0} \rightarrow \boldsymbol{\Delta}^{1}$ denote the inclusions of the initial and terminal endpoints of the interval. If $\mathbf{S}$ is a simplicial set, we also denote by $\boldsymbol{\lambda}_{i}: \mathbf{S} \rightarrow \mathbf{S} \times \boldsymbol{\Delta}^{1}$ the simplicial maps $\mathbf{S} \times \boldsymbol{\lambda}_{i}$. If $(\mathbf{E}, \mathbf{p}) \in \mathcal{S}_{\mathbf{K}}$, we abuse notation and denote by $\mathbf{p}: \mathbf{E} \times \boldsymbol{\Delta}^{1} \rightarrow \mathbf{K}$ the composite $\mathbf{E} \times \boldsymbol{\Delta}^{1} \rightarrow \mathbf{E} \stackrel{\mathbf{p}}{\rightarrow} \mathbf{K}$.

Definition 5.6. If $\boldsymbol{f}_{0}, \boldsymbol{f}_{1}:(\mathbf{E}, \mathbf{p}) \rightarrow\left(\mathbf{E}^{\prime}, \mathbf{p}^{\prime}\right)$ are two $\mathcal{S}_{\mathbf{K}}$ morphisms and $\left(\mathbf{E}^{\prime}, \mathbf{p}^{\prime}\right)$ is a Kan fibration, then $\boldsymbol{f}_{0}$ is homotopic to $\boldsymbol{f}_{1}$ over $\mathbf{K}$ if there exists an $\mathcal{S}_{\mathbf{K}}$ morphism $\boldsymbol{F}:\left(\mathbf{E} \times \boldsymbol{\Delta}^{1}, \mathbf{p}\right) \rightarrow\left(\mathbf{E}^{\prime}, \mathbf{p}^{\prime}\right)$, such that $\boldsymbol{F} \circ \boldsymbol{\lambda}_{i}=\boldsymbol{f}_{i}, i=0,1$. We write $\boldsymbol{f}_{0} \sim_{\mathbf{K}} \boldsymbol{f}_{1}$.

The set of homotopy classes of morphisms [16, page 28] $\boldsymbol{f} \in \mathcal{S}_{\mathbf{K}}\left((\mathbf{E}, \mathbf{p}),\left(\mathbf{E}^{\prime}, \mathbf{p}^{\prime}\right)\right)$, is denoted by $\left[(\mathbf{E}, \mathbf{p}),\left(\mathbf{E}^{\prime}, \mathbf{p}^{\prime}\right)\right]$. We use an argument of Goyo [12] to prove:

Theorem 5.7. Let $(\mathbf{E}, \mathbf{p})$ be a Kan fibration and suppose $\left(A \otimes_{R} \Lambda Y, D\right)$ is a 1connected A minimal model of finite type. Then the bijection of Theorem 5.4 induces a bijection $\left[(\mathbf{E}, \mathbf{p}),\left\langle A \otimes_{R} \Lambda Y\right\rangle\right] \cong\left[A \otimes_{R} \Lambda Y, \mathcal{F}(\mathbf{E}, \mathbf{p})\right]$ (see Section 4).

Proposition 5.8. (i) If $f_{0} \sim_{\mathbf{K}} f_{1}:\left(A \otimes_{R} \Lambda Y, D\right) \rightarrow\left(E, j_{E}\right)$ are homotopic morphisms from a 1-connected A minimal model $\left(A \otimes_{R} \Lambda Y, D\right)$, then $\left\langle f_{0}\right\rangle \sim_{\mathbf{K}}\left\langle f_{1}\right\rangle$ : $\left\langle E, j_{E}\right\rangle \rightarrow\left\langle A \otimes_{R} \Lambda Y, D\right\rangle$.

(ii) If $\mathbf{f}_{0} \sim_{\mathbf{K}} \mathbf{f}_{1}:(\mathbf{E}, \mathbf{p}) \rightarrow\left(\mathbf{E}^{\prime}, \mathbf{p}^{\prime}\right)$ are homotopic $\mathcal{S}_{\mathbf{K}}$ morphisms, with $\left(\mathbf{E}^{\prime}, \mathbf{p}^{\prime}\right) \in$ $\mathcal{K}_{\mathbf{K}}^{f}$, then $\mathcal{F}\left(\mathbf{f}_{0}\right) \sim_{\mathbf{K}} \mathcal{F}\left(\mathbf{f}_{1}\right): \mathcal{F}\left(\mathbf{E}^{\prime}, \mathbf{p}^{\prime}\right) \rightarrow \mathcal{F}(\mathbf{E}, \mathbf{p})$.

Proof. (i) The $f_{i}$ factor as $f_{i}=\varepsilon_{i} \circ F$, where $F:\left(A \otimes_{R} \Lambda Y, D\right) \rightarrow E \otimes_{\mathbf{k}} \Lambda(t, d t)$. Thus $\left\langle f_{i}\right\rangle=\langle F\rangle \circ\left\langle\varepsilon_{i}\right\rangle$. But it is immediate from the definition that $\left\langle E \otimes_{\mathbf{k}} \Lambda(t, d t)\right\rangle=$ $\langle E\rangle \times\langle\Lambda(t, d t)\rangle$. It is thus sufficient to prove $\langle\Lambda(t, d t)\rangle$ is contractible.

A simplicial map $\psi: \partial \boldsymbol{\Delta}^{n} \rightarrow\langle\Lambda(t, d t)\rangle$ determines, by adjunction, a morphism $\varphi: \Lambda(t, d t) \rightarrow A\left(\boldsymbol{\partial} \boldsymbol{\Delta}^{n}\right)$, which lifts to a morphism $\Lambda(t, d t) \rightarrow A\left(\boldsymbol{\Delta}^{n}\right)$ since $A(*)$ is 
extendable. Thus $\psi$ extends to $\bar{\psi}: \boldsymbol{\Delta}^{n} \rightarrow\langle\Lambda(t, d t)\rangle$, and $\psi$ is homotopically trivial. Thus the Kan complex $\langle\Lambda(t, d t)\rangle$ having trivial homotopy groups is contractible.

(ii) Let $\boldsymbol{\pi}: \mathbf{E} \times \boldsymbol{\Delta}^{1} \rightarrow \mathbf{E}$ be the projection and $m: A \otimes_{R} \Lambda Y \stackrel{\simeq}{\rightrightarrows} \mathcal{F}\left(\mathbf{E}^{\prime}, \mathbf{p}^{\prime}\right)$ be a minimal model. Then the quasi-isomorphisms $\mathcal{F}(\boldsymbol{\pi}), \mathcal{F}\left(\boldsymbol{\lambda}_{0}\right), \mathcal{F}\left(\boldsymbol{\lambda}_{1}\right)$ induce bijections $\mathcal{F}(\boldsymbol{\pi})_{\sharp}, \mathcal{F}\left(\boldsymbol{\lambda}_{0}\right)_{\sharp}$ and $\mathcal{F}\left(\boldsymbol{\lambda}_{1}\right)_{\sharp}$ in $\left[A \otimes_{R} \Lambda Y,-\right]$. Since $\boldsymbol{\pi} \boldsymbol{\lambda}_{i}=$ identity, it follows that $\mathcal{F}\left(\boldsymbol{\lambda}_{0}\right)_{\sharp}=\mathcal{F}(\boldsymbol{\pi})_{\sharp}^{-1}=\mathcal{F}\left(\boldsymbol{\lambda}_{1}\right)_{\sharp}$. If $\mathbf{F}:\left(\mathbf{E} \times \boldsymbol{\Delta}^{1}, \mathbf{p}\right) \rightarrow\left(\mathbf{E}^{\prime}, p^{\prime}\right)$ is a homotopy from $\boldsymbol{f}_{0}$ to $\boldsymbol{f}_{1}$, then we have $\left[\mathcal{F}\left(\boldsymbol{f}_{1}\right) \circ m\right]=\mathcal{F}\left(\boldsymbol{\lambda}_{1}\right)_{\sharp}[\mathcal{F}(\mathbf{F}) \circ m]=\mathcal{F}\left(\boldsymbol{\lambda}_{0}\right)_{\sharp}[\mathcal{F}(\mathbf{F}) \circ m]=\left[\mathcal{F}\left(\boldsymbol{f}_{0}\right) \circ m\right]$. Proposition 4.5 implies $\left[\mathcal{F}\left(\boldsymbol{f}_{0}\right)\right]=\left[\mathcal{F}\left(\boldsymbol{f}_{1}\right)\right]$.

Proof of Theorem 5.7. First, suppose $f_{0}, f_{1}: A \otimes_{R} \Lambda Y \rightarrow \mathcal{F}(\mathbf{E}, \mathbf{p})$ are homotopic maps. Decompose their adjoints $\boldsymbol{f}_{0}, \boldsymbol{f}_{1}:(\mathbf{E}, \mathbf{p}) \rightarrow\left\langle A \otimes_{R} \Lambda Y\right\rangle$ as $(\mathbf{E}, \mathbf{p}) \rightarrow$ $\langle\mathcal{F}(\mathbf{E}, \mathbf{p})\rangle \stackrel{\left\langle f_{i}\right\rangle}{\rightarrow}\left\langle A \otimes_{R} \Lambda Y\right\rangle$. Proposition 5.8 now implies that $\boldsymbol{f}_{0} \sim_{\mathbf{K}} \boldsymbol{f}_{1}$.

For the reverse implication let $\boldsymbol{f}_{0}, \boldsymbol{f}_{1}:(\mathbf{E}, \mathbf{p}) \rightarrow\left\langle A \otimes_{R} \Lambda Y\right\rangle$ be two simplicial maps, homotopic over K. Their adjoints factor as $A \otimes_{R} \Lambda Y \rightarrow \mathcal{F}\left\langle A \otimes_{R} \Lambda Y\right\rangle^{\mathcal{F}\left(f_{i}\right)} \mathcal{F}(\mathbf{E}, \mathbf{p})$, and the $\mathcal{F}\left(\boldsymbol{f}_{i}\right)$ are homotopic by Proposition 5.8 .

If $(\mathbf{E}, \mathbf{p})$ is a Kan fibration in $\mathcal{K}_{\mathbf{K}}^{f}$, then we can apply Theorem 3.12 to obtain the (unique) minimal model $\left(A \otimes_{R} \Lambda Y, D\right)$ of $\mathcal{F}(\mathbf{E}, \mathbf{p})$ together with a quasi-isomorphism $m:\left(A \otimes_{R} \Lambda Y, D\right) \stackrel{\simeq}{\longrightarrow} \mathcal{F}(\mathbf{E}, \mathbf{p})$. This corresponds under the bijection of Theorem 5.4 to a morphism of Kan fibrations over $\mathbf{K}, \mathbf{m}:(\mathbf{E}, \mathbf{p}) \rightarrow\left\langle\left(A \otimes_{R} \Lambda Y, D\right), j\right\rangle$.

Let $\mathbf{F}$ be the fibre of $(\mathbf{E}, \mathbf{p})$ and let $(\Lambda Z, d)$ be a representative Sullivan algebra, so that $m_{v}:(\Lambda Z, d) \stackrel{\simeq}{\rightrightarrows} A(\mathbf{F})$. By hypothesis, $\mathbf{F}$ is simply connected. Since $A(1)$ is concentrated in degrees 1 and 0 and since $Z=Z^{\geq 2},\langle\Lambda Z, d\rangle$ is even 2-reduced. Since both simplicial sets are Kan complexes, $\pi_{n}(\mathbf{F})=\left[\boldsymbol{\partial} \boldsymbol{\Delta}^{n+1}, \boldsymbol{F}\right]$ and $\pi_{n}\langle\Lambda Z, d\rangle=$ $\left[\boldsymbol{\partial} \boldsymbol{\Delta}^{n+1},\langle\Lambda Z, d\rangle\right]$. Since $(\Lambda Z, d)$ is minimal, $\left[(\Lambda Z, d), A\left(\boldsymbol{\partial} \boldsymbol{\Delta}^{n+1}\right)\right]=\operatorname{Hom}\left(Z^{n}, \mathbf{k}\right)$.

Thus $\mathbf{m}^{\mathbf{v}}$ and $m_{v}$ determine the commutative diagram

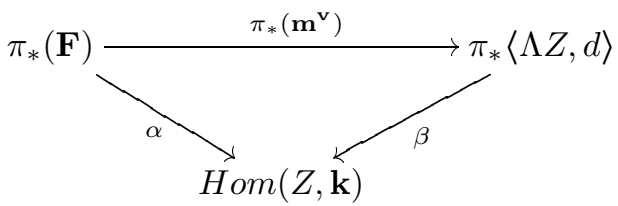

It is an easy exercise that $\beta$ is a group homomorphism (and hence, by Theorem 5.7 an isomorphism) compatible with the structures of $\pi_{1}(\mathbf{K})$-modules (4.6). This identifies $\pi_{*}\langle\Lambda Z, d\rangle$ as a graded $\mathbf{k}$ vector space. On the other hand, since $m_{v}$ is a quasi-isomorphism, the classical Postnikov tower argument [21, bottom of page 309], using the fact that $Z$ has finite type, shows that $\alpha$ extends to an isomorphism $\pi_{*}(\mathbf{F}) \otimes_{\mathbb{Z}} \mathbf{k} \cong \operatorname{Hom}(Z, \mathbf{k})$.

In summary then we have

Proposition 5.9. The map $\pi_{*}\left(\mathbf{m}^{\mathbf{v}}\right)$ extends uniquely to an isomorphism $\pi_{*}\left(\mathbf{m}^{\mathbf{v}}\right)$ : $\pi_{*}(\mathbf{F}) \otimes_{\mathbb{Z}} \mathbf{k} \stackrel{\cong}{\longrightarrow} \pi_{*}\langle\Lambda Z, d\rangle$, while $m_{v}$ induces an isomorphism $H\left(m_{v}\right): H(\Lambda Z, d) \stackrel{\cong}{\longrightarrow}$ $H^{*}(\mathbf{F} ; \mathbf{k})$ of algebras and $\pi_{1}(\mathbf{K})$-modules.

For the rest of this section, $\mathbf{k}=\mathbb{Q}$. As usual we put $R(*)=A(*)^{0}$.

Denote by $\mathcal{K}_{\mathbf{K}}^{f, 0}$ the full subcategory of $\mathcal{K}_{\mathbf{K}}^{f}$ in which the homotopy groups of the fibre are rational vector spaces. We use the two functors \langle\rangle and $\mathcal{F}$ to construct natural equivalences of homotopy categories connecting $\mathcal{K}_{\mathbf{K}}^{f, 0}$ and $\mathcal{M}_{\mathbf{K}}$. A version over $\mathbb{R}$ using $A_{D R}(*)$ and continuous cohomology also exists [21, Theorem (8.1)']. 
If $\left(A \otimes_{R} \Lambda Y, D\right)$ is any minimal model in the subcategory $\mathcal{M}_{\mathbf{K}}$, the identity map of $\left\langle\left(A \otimes_{R} \Lambda Y, D\right), j\right\rangle$ corresponds under the bijection of Theorem 5.4 to a morphism

$$
\Theta:\left(A \otimes_{R} \Lambda Y, D\right) \rightarrow \mathcal{F}\left(\left\langle\left(A \otimes_{R} \Lambda Y, D\right), j\right\rangle\right) .
$$

Theorem 5.10. Let $(\mathbf{E}, \mathbf{p})$ be a Kan fibration in $\mathcal{K}^{f}$ and let $\left(A \otimes_{R} \Lambda Y, D\right)$ be a minimal model in $\mathcal{M}_{\mathbf{K}}$. Then

(i) $\mathbf{m}:(\mathbf{E}, \mathbf{p}) \rightarrow\left\langle\left(A \otimes_{R} \Lambda Y, D\right), j\right\rangle$ is a fibrewise rationalisation in the sense of Bousfield and Kan [2, Chapter 1, §8]; i.e., its restriction to the typical fibre is a rationalisation. In particular, if $(\mathbf{E}, \mathbf{p}) \in \mathcal{K}^{f, 0}$, then $\mathbf{m}$ is a fibrewise homotopy equivalence.

(ii) $\Theta$ is a quasi-isomorphism of local systems, and hence a minimal model.

Proof. (i) It follows from Proposition 5.9 that $\mathbf{m}$ is a homotopy equivalence. Thus by the simplicial version of a theorem of Dold [7, $\mathbf{m}$ is a fibrewise homotopy equivalence.

(ii) Since both the domain and target of $\Theta$ are $A$-algebras, it is sufficient to verify that $\Theta_{v}$ is a quasi-isomorphism of DGA's. Let $(\Lambda Z, d)$ be a representative Sullivan model for $\left(A \otimes_{R} \Lambda Y, D\right)$. Then $\Theta_{v}$ is the DGA morphism $(\Lambda Z, d) \rightarrow A(\langle\Lambda Z, d\rangle)$ adjoint to the identity map of $\langle\Lambda Z, d\rangle$. Thus it is a quasi-isomorphism by [21] Theorem 8.1].

Suppose, finally, that in a category $\mathcal{C}$ there is specified an equivalence relation, homotopy, in each morphism set $\mathcal{C}\left(C, C^{\prime}\right)$, compatible with composition, and recall that the associated homotopy category, ho- $\mathcal{C}$, is the category with the same objects, but with morphisms ho- $\mathcal{C}\left(C, C^{\prime}\right)$ the set of homotopy classes of $\mathcal{C}$ morphisms from $C$ to $C^{\prime}$. A homotopy equivalence in $\mathcal{C}$ is a morphism that becomes an isomorphism in ho- $\mathcal{C}$. Thus the homotopy equivalences in $\mathcal{K}^{f, 0}$ are precisely the fibrewise homotopy equivalences. Exactly as in the case when $\mathbf{K}=\mathbf{p t}$ we have [21 Theorem 10.1]:

Theorem 5.11. The functor \langle\rangle$: \mathcal{M}_{\mathbf{K}} \leadsto \mathcal{K}_{\mathbf{K}}^{f, 0}$ induces a natural equivalence of homotopy categories.

\section{NON-SIMPLY CONNECTED RATIONAL HOMOTOPY THEORY}

In this section we work over the ground field $\mathbb{Q}$ and $A(*)$ is an admissible simplicial CDGA over $\mathbb{Q}$ (cf. Section 3). We apply the results of Section 5 to classify non-simply connected rational homotopy types.

Definition 6.1. A path connected topological space, $X$, is rational if its universal cover $\tilde{X}$ is; i.e. if $\pi_{i}(X)$ is a rational vector space for $i \geq 2$. The rationalisation $X_{\mathbb{Q}}$ of $X$ is the fibrewise rationalisation of the fibration $\tilde{X} \rightarrow X \rightarrow K\left(\pi_{1}(X), 1\right)$. The rational homotopy type of $X$ is the homotopy type of its rationalisation, $X_{\mathbb{Q}}$.

Let $\mathcal{T}$ be the category of pointed path connected topological spaces $X$. Let $\mathcal{T}^{f}$ be the full subcategory of spaces $X$ such that $\pi_{i}(X) \otimes \mathbb{Q}$ is finite dimensional for $i \geq 2$ (equivalently $H_{i}(\tilde{X} ; \mathbb{Q})$ is finite dimensional for $i \geq 2$ ), and let $\mathcal{T}^{f, 0}$ be the full subcategory of rational spaces in $\mathcal{T}^{f}$. Two maps in $\mathcal{T}$ (or in $\mathcal{T}^{f}$ or $\mathcal{T}^{f, 0}$ ) are homotopic if they are connected by a basepoint-preserving homotopy. Denote by $\mathcal{T}_{C W}, \mathcal{T}_{C W}^{f}$ and $\mathcal{T}_{C W}^{f, 0}$ the full subcategories of spaces of the homotopy type of a CW-complex. 
Theorem 6.2. (i) There is an algebraic category $\mathcal{M}$ with a relation of homotopy in its morphism sets, and there is a homotopy preserving functor from $\mathcal{M}$ to the category $\mathcal{T}_{C W}^{f, 0}$ of rational spaces, inducing an equivalence of homotopy categories: $h o-\mathcal{M} \simeq h o-\mathcal{T}_{C W}^{f, 0}$.

(ii) In $\mathcal{M}$ the homotopy equivalences are isomorphisms.

Proof. In 8] I.3] Eilenberg and Mac Lane describe a classifying functor $\pi \hookrightarrow \mathbf{B} \pi$ from the category of discrete groups to the category of 1-reduced simplicial $K(\pi, 1)$ 's. This functor is an isomorphism onto a full subcategory of Kan complexes with inverse the functor $\pi_{1}$. We denote this subcategory by $\mathcal{T}(1)$.

Now let $\mathcal{K}$ denote the category whose objects are the 1-reduced Kan fibrations with simply connected fibre and with base some $\mathbf{K} \in \mathcal{T}(1)$ and whose morphisms are the commutative diagrams

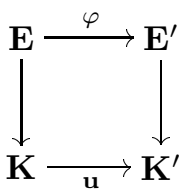

of simplicial maps. We denote by $\mathcal{K}^{f}$ the full subcategory of $\mathcal{K}$ in which the rational homology groups of the fibre are all finite dimensional, and by $\mathcal{K}^{f, 0}$ the full subcategory of $\mathcal{K}^{f}$ in which the homotopy groups of the fibre are themselves rational vector spaces. A morphism $(\varphi, \mathbf{u}): \mathbf{E} \rightarrow \mathbf{E}^{\prime}$ in $\mathcal{K}\left(\right.$ or $\mathcal{K}^{f}$ or $\mathcal{K}^{f, 0}$ ) factors as $\mathbf{E} \stackrel{\varphi^{\mathbf{u}}}{\rightarrow}\left(\mathbf{E}^{\prime}\right)^{\mathbf{u}} \rightarrow \mathbf{E}^{\prime}$ through the pull-back $\left(\mathbf{E}^{\prime}\right)^{\mathbf{u}}$. Two morphisms $(\boldsymbol{\varphi}, \mathbf{u})$ and $(\boldsymbol{\psi}, \mathbf{w})$ are homotopic if $\mathbf{u}=\mathbf{w}$ and $\varphi^{\mathbf{u}} \sim_{\mathbf{K}} \boldsymbol{\psi}^{\mathbf{u}}$ (cf. Section 5).

Denote by $\operatorname{Sing}_{1}$ the functor from pointed topological spaces to 1-reduced simplicial sets that assigns to $X$ the 1-reduced singular simplices on $X$. In [ㅁ, II.7] this is extended to the functor $\widetilde{\operatorname{Sing}}{ }_{1}: \mathcal{T} \leadsto \mathcal{K}$ that assigns to $X$ the Kan fibration $\operatorname{Sing}_{1}(X) \stackrel{\boldsymbol{\rho}}{\rightarrow} \mathbf{B} \boldsymbol{\pi}_{1}(X)$ defined as follows: if $\sigma:\left\langle e_{0}, \ldots, e_{n}\right\rangle \rightarrow X$ is a 1-reduced $n$ simplex, then $\boldsymbol{\rho} \sigma=\left(g_{1}, \cdots, g_{n}\right)$ with $g_{i} \in \pi_{1}(X)$ represented by the restriction of $\sigma$ to the edge $\left\langle e_{i-1}, e_{i}\right\rangle$. On the other hand, if || denotes the Milnor realisation functor, then it can be considered as a functor ||$: \mathcal{K} \leadsto \mathcal{T}$ assigning to $\mathbf{E} \rightarrow \mathbf{K}$ the pointed CW complex $|\mathbf{E}|$.

It is straightforward to check that || and $\widetilde{\operatorname{Sing}}{ }_{1}$ are adjoint functors and preserve the relation of homotopy for $\mathcal{T}$ and $\mathcal{K}$ as described above. A small variation on the classical result for Sing and || shows that the functors || and $\widetilde{\operatorname{Sing}_{1}}$ induce natural equivalences of homotopy categories: ho- $\mathcal{K} \simeq h o-\mathcal{T}_{C W}, h o-\mathcal{K}^{f} \simeq$ ho- $\mathcal{T}_{C W}^{f}$ and $h o-\mathcal{K}^{f, 0} \simeq h o-\mathcal{T}_{C W}^{f, 0}$.

We now define the category $\mathcal{M}$ as follows:

- the objects of $\mathcal{M}$ are pairs $\left(E_{\mathbf{K}}, \mathbf{K}\right)$ with $\mathbf{K}$ in $\mathcal{T}(1)$ and $E_{\mathbf{K}}$ in $\mathcal{M}_{\mathbf{K}}(\S 5)$.

- the morphisms $\mathcal{M}\left(E_{\mathbf{K}}, E_{\mathbf{K}^{\prime}}^{\prime}\right)$ are the pairs $(\varphi, \mathbf{u})$ in which $\mathbf{u}: \mathbf{K} \rightarrow \mathbf{K}^{\prime}$ is a simplicial map and $\varphi: E_{\mathbf{K}} \leftarrow\left(E_{\mathbf{K}^{\prime}}^{\prime}\right)^{\mathbf{u}}$ an $A_{\mathbf{K}}$-morphism from the pull-back $\left(E_{\mathbf{K}^{\prime}}^{\prime}\right)^{\mathbf{u}}$ to $E_{\mathbf{K}}$. Two morphisms $(\varphi, \mathbf{u})$ and $(\psi, \mathbf{w})$ are homotopic if $\mathbf{u}=\mathbf{w}$ and $\varphi \sim_{\mathbf{K}} \psi$.

The functor \langle\rangle$: \mathcal{M}_{\mathbf{K}} \leadsto \mathcal{K}_{\mathbf{K}}^{f, 0}$ of Section 5 defines a functor \langle\rangle$: \mathcal{M} \leadsto \mathcal{K}^{f, 0}$ which preserves homotopy, and it follows from Theorem 5.11 that this induces an equivalence of categories, $h o-\mathcal{M} \simeq h o-\mathcal{K}^{f, 0}$. The yields a natural equivalence $h o-\mathcal{M} \simeq h o-\mathcal{T}_{C W}^{f, 0}$. Finally, it follows at once from Theorem 3.7 that the homotopy equivalences in $\mathcal{M}$ are isomorphisms. 
Fix a pointed topological space $X$ in $\mathcal{T}^{f}$ and denote the local system $A_{\mathbf{B} \pi_{1}(X)}$

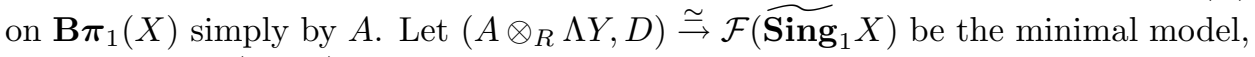
and denote by $(\Lambda Z, d)$ its representative Sullivan algebra.

Definition 6.3. The minimal model $\left(A \otimes_{R} \Lambda Y, D\right)$ will be called the minimal model of the space $X$.

As in Theorem 5.10 we have

Theorem 6.4. The realisation functor $\left|\left\langle A \otimes_{R} \Lambda Y, D\right\rangle\right|$ is a rationalisation of the space $X$.

As in the classical case, it follows from Theorem 6.4 that any invariant of the rational homotopy type of $X$ can be read off from its minimal model, and that any invariant of the isomorphism class of the model is a rational homotopy type invariant for $X$. Giving an explicit description is an entirely different matter however. For the classical invariants of homology and homotopy we have:

Theorem 6.5. Let $\left(A \otimes_{R} \Lambda Y, D\right)$ be the minimal model of a space $X$ in $\mathcal{T}^{f}$, and let $(\Lambda Z, d)$ be a representative Sullivan algebra. Then

(i) The base $\mathbf{K}$ of $\left(A \otimes_{R} \Lambda Y, D\right)$ is just $\mathbf{B} \pi_{1}(X)$ so that $\pi_{1}(X)=\pi_{1}(\mathbf{K})$.

(ii) The $C D G A,(\Lambda Z, d)$, is the minimal Sullivan algebra of the universal cover, $\tilde{X}$, of $X$. In particular, $\pi_{k}(X) \otimes \mathbb{Q} \cong \operatorname{Hom}\left(Z^{k}, \mathbb{Q}\right), k \geq 2$, and $H^{*}(\tilde{X} ; \mathbb{Q}) \cong$ $H(\Lambda Z, d)$ as graded algebras. Moreover these two isomorphisms are isomorphisms of $\pi_{1}(X)$-modules ( $c f$. 4.6).

(iii) The $D G A$ 's $C^{*}(X, \mathbb{Q})$ and $\Gamma\left(A \otimes_{R} \Lambda Y, D\right)$ are connected by a sequence of DGA quasi-isomorphisms. Thus there is an isomorphism of graded algebras, $H^{*}(X ; \mathbb{Q}) \cong H \Gamma\left(A \otimes_{R} \Lambda Y, D\right)$.

Proof. (i) is by definition and, as we remarked just before Proposition 5.9, (ii) is standard [21, §8]. For (iii) recall from [13, Theorem 12.27] that the quasiisomorphism of local systems induces a DGA quasi-isomorphism $\Gamma\left(A \otimes_{R} \Lambda Y, D\right) \stackrel{\simeq}{\longrightarrow}$ $\Gamma \mathcal{F}\left(\operatorname{Sing}_{1}(X), \boldsymbol{\rho}\right)$. As in [13 Lemma 19.21] the latter DGA is isomorphic to $A\left(\operatorname{Sing}_{1}(X)\right)$, while as in [13, Proof of Theorem 14.18] $A\left(\mathbf{S i n g}_{1}(X)\right)$ is connected to $C^{*}(X)$ by a chain of DGA quasi-isomorphisms.

Example 6.6. $\pi_{1}=\mathbb{Z},(\Lambda Z, d)$ arbitrary.

Here $\mathbf{B} \pi_{1} \simeq S^{1}$ and the fibration $\tilde{X} \rightarrow X \rightarrow S^{1}$ is determined by the action of the generator $g$ of $\mathbb{Z}$ on $\tilde{X}$, or, equivalently, by the single automorphism $\rho(g)$ of $(\Lambda Z, d)$. Thus in this case the representation lifts to a representation in $\operatorname{Aut}(\Lambda Z, d)$ which determines $X$.

Example 6.7. $\pi_{1}=\mathbb{Z} \times \mathbb{Z},(\Lambda Z, d)=(\Lambda(x, y, z, w, u), d)$ with $x, y, z$ cocycles of degree $3, w$ a cocycle of degree $6, \operatorname{deg} u=5, d u=y z$.

Here $\mathbf{B} \pi_{1} \simeq S^{1} \times S^{1}$. Thus a fibration over $\mathbf{B} \pi_{1}$ with fibre $\tilde{X}$ is described by

- two automorphisms $\varphi$ and $\psi$ of $(\Lambda Z, d)$, which together define the fibration over $S^{1} \vee S^{1}$.

- a homotopy $\varphi \psi \sim \psi \varphi$, which permits the extension of this fibration to a fibration over $S^{1} \times S^{1}$.

Consider the automorphisms $\varphi$ and $\psi$ given by:

$\varphi$ is the identity on each generator but $w$, while $\varphi(w)=w+x y$;

$\psi$ is the identity on each generator but $x$, while $\psi(x)=x+z$. 
Then $\varphi \circ \psi$ is homotopic to $\psi \circ \varphi$ via the morphism $F:(\Lambda Z, d) \rightarrow(\Lambda Z, d) \otimes \Lambda(t, d t)$, which is the identity on each generator but $x$ and $w$, and is given there by $F(x)=$ $x+z$ and $F(w)=w+x y+t z y-d t \wedge u$. Thus $\varphi$ and $\psi$ determine a fibration $\tilde{X} \rightarrow X \rightarrow S^{1} \times S^{1}$.

The induced representation of $\mathbb{Z} \times \mathbb{Z}$ in the cohomology $H(\Lambda Z, d)$ of the fibre is exactly that given by $H(\varphi)$ and $H(\psi)$. It is a straightforward calculation that for any morphisms $\varphi^{\prime}$ and $\psi^{\prime}$ inducing respectively $H(\varphi)$ and $H(\psi)$ we have $\varphi^{\prime} \psi^{\prime} \neq$ $\psi^{\prime} \varphi^{\prime}$. Thus this representation in $H(\Lambda Z, d)$ does not lift to a representation in Aut $(\Lambda Z, d)$. On the other hand, since $(\Lambda Z, d)=\left(\Lambda Z, d_{2}\right)$, the fibration does furnish us with a representation of $\pi_{1}$ in $(\Lambda Z, d)$. However this representation does not lift the canonical representation of $\pi_{1}$ in $\pi_{0} A u t(\Lambda Z, d)$

Next observe that the representation of $\mathbb{Z} \times \mathbb{Z}$ in $H^{*}(X ; \mathbb{Q})$ is nilpotent. Hence $X$ is a nilpotent space, and there exists a classical minimal model which contains the rational homotopy type of the fibration $\tilde{X} \rightarrow X \rightarrow S^{1} \times S^{1}$. A computation shows this has the form: $(\Lambda(\alpha, \beta), 0) \rightarrow(\Lambda(\alpha, \beta) \otimes \Lambda Z, D) \rightarrow(\Lambda Z, d)$, where $\operatorname{deg} \alpha=$ $\operatorname{deg} \beta=1, D y=D z=0, D x=\beta z, D w=\alpha x y-\alpha \beta u, D u=y z$.

Example 6.8. $\pi_{1}=\mathbb{Z} \times \mathbb{Z} \times \mathbb{Z} \times \mathbb{Z},(\Lambda Z, d)=(\Lambda(u, v), d)$ with $u$ a cocycle of degree $2, \operatorname{deg} v=3, d v=u^{2}$.

Here $\mathbf{B} \boldsymbol{\pi}_{1} \simeq S^{1} \times S^{1} \times S^{1} \times S^{1}$. Let $A(*)=A_{P L}(* ; \mathbb{Q})$ and $A=A_{\mathbf{B} \pi_{1}}$. An $A$ minimal model $\left(A \otimes_{R} \Lambda Z, D\right)$ is specified by setting $(D u)_{\sigma}=0$ and $(D v)_{\sigma}=u^{2}+z_{\sigma}$, $\sigma \in \mathbf{B} \boldsymbol{\pi}_{1}$, where $\left\{z_{\sigma}\right\} \in A^{4}\left(\mathbf{B} \pi_{1}\right)$ is a cocyle representing the fundamental class of $S^{1} \times S^{1} \times S^{1} \times S^{1}$.

The resulting space $X=\left\langle A \otimes_{\mathbb{Q}} \Lambda Z, D\right\rangle$ satisfies $\tilde{X} \simeq S_{\mathbb{Q}}^{2}$ and $\pi_{1}(X)=\mathbb{Z} \times$ $\mathbb{Z} \times \mathbb{Z} \times \mathbb{Z}$. The action of $\pi_{1}$ on $\pi_{*}(\tilde{X})$ and on $H_{*}(\tilde{X})$ is trivial; however $X \not$ $S_{\mathbb{Q}}^{2} \times S^{1} \times S^{1} \times S^{1} \times S^{1}$, and so the trivial representation does not determine $X$. As In Example 6.7 $X$ is a nilpotent space. Its minimal Sullivan model is $(\Lambda(\alpha, \beta, \gamma, \delta, u, v), D)$ with $D=0$ on all the generators but $v$ and $D v=u^{2}+\alpha \beta \gamma \delta$.

\section{Applications, examples And PROBlems}

In this section, we work over a fixed field $\mathbf{k}$ of characteristic zero; $A(*)$ is an admissible simplicial CDGA with coefficients in $\mathbf{k}$. Classical minimal Sullivan algebras will be 1-connected and of finite type unless we explicitly assert the contrary.

7.1. Free commutative cochain algebras. Consider commutative cochain algebras, $(B, \delta)$, in which the underlying algebra is connected, free and of finite type: $B=\Lambda V, V=\left\{V^{i}\right\}_{i>1}$ and $\operatorname{dim} V^{i}<\infty, i \geq 1$. Suppose further that $\operatorname{Im} \delta \subset B^{+} . B^{+}$. Then necessarily $\left(\Lambda V^{1}, \delta\right)$ is a sub cochain algebra. More generally suppose given some $V^{1} \subset W \subset V$ such that $(\Lambda W, \delta)$ is a sub cochain algebra. Write $V=W \oplus Z$ so that $B=\Lambda W \otimes \Lambda Z$, and consider the sequence of cochain algebra morphisms: $\mathcal{E}(\Lambda W, \delta) \rightarrow(\Lambda W \otimes \Lambda Z, \delta) \stackrel{\rho}{\rightarrow}(\Lambda Z, d)$, where $\rho=0$ in $W$ and $\rho=i d$ in $Z$. It may happen that $Z$ can be written as the increasing union of graded subspaces $Z(k), k \geq 0$, such that $\delta: Z(0) \rightarrow \Lambda W$ and $\delta: Z(k) \rightarrow \Lambda W \otimes \Lambda Z(k-1)$, $k \geq 1$. In this case the sequence $\mathcal{E}$ is called generalized nilpotent [21] or a relative Sullivan algebra [10].

A local system, $(E, D)$, of commutative cochain algebras on $\langle\Lambda W, \delta\rangle$ is defined by $\left(E_{\sigma}, D_{\sigma}\right)=A(n) \otimes_{\sigma}(\Lambda W \otimes \Lambda Z, \delta)$. Put $A=A_{\langle\Lambda W, \delta\rangle}$. 
Proposition 7.1. (i) If either $\mathcal{E}$ is generalized nilpotent, or if $\mathbf{k}=\mathbb{R}$ and $A(*)=$ $A_{D R}(*)$, then $(E, D)$ is an A minimal model.

(ii) In either case the realisation $\langle E, D\rangle$ of $\S 5$ coincides with Sullivan's realisation $\langle\Lambda W \otimes \Lambda Z, \delta\rangle$.

Remark 7.2. If $\mathcal{M}=(\Lambda V, \delta)$ is a connected cochain algebra of finite type with $\operatorname{Im} \delta \subset \Lambda^{+} V \cdot \Lambda^{+} V$, then we have the sequence $\left(\Lambda V^{1}, \delta\right) \rightarrow(\Lambda V, \delta) \rightarrow\left(\Lambda V^{\geq 2}, d\right)$. In this case, when $\mathbf{k}=\mathbb{Q}$ and $A(*)=A_{P L}(*)$, Propositions [5.5 and 5.9 reduce to a result of Sullivan [21, Theorem 8.1]. When $\mathbf{k}=\mathbb{R}$ and $A(*)=A_{D R}(*)$, these propositions reduce to part of [21, Theorem $\left.8.1^{\prime}\right]$.

Remark 7.3. If $\mathbf{k}=\mathbb{Q}$ as opposed to $\mathbf{k}=\mathbb{R}$, it is necessary to impose the condition of generalized nilpotence in 7.1. In fact, consider $\mathcal{M}=(\Lambda(x, y), d)$, where $\operatorname{deg} x=$ 3 , deg $y=1, d y=0, d x=y x$. For the 1-simplex $\sigma: \mathcal{M}^{1} \rightarrow \Lambda(t, d t)$, defined by $\sigma(y)=d t$, the face operator $\Lambda(t, d t) \otimes_{\sigma} \mathcal{M} \rightarrow \mathcal{M}, t \mapsto 0$, is not a quasi-isomorphism. Thus $\mathcal{M}$ is not an $A$ minimal model.

Remark 7.4. In the minimal model of Proposition 7.1 the fibre $\left(\Lambda Y, D_{0}\right)$ is given by $\left(\Lambda Y, D_{0}\right)_{\sigma}=A^{0}(n) \otimes_{\sigma}(\Lambda W \otimes \Lambda Z, \delta)=A^{0}(n) \otimes_{\mathbf{k}}(\Lambda Z, d)$, where we identify $A^{0}(n)=A(n) / A^{+}(n)$. Thus the fibre is the free $A^{0}$-module, $A^{0} \otimes_{\mathbf{k}}(\Lambda Z, d)$. (Note that as shown by Examples 6.6 and 6.7 this does not imply that $\pi_{1}\langle\Lambda W, \delta\rangle$ acts trivially in $H(\Lambda Z, d)$.)

For general $A$ minimal models, however, the fibre $(\Lambda Y, \bar{D})$ will not be $A^{0}$-free. For example, suppose $\mathbf{k}=\mathbb{R}$ and consider the representation of $\mathbb{Z}_{2}$ in $(\Lambda x, 0)$, $\operatorname{deg} x=3$, given by $1 \circ x=-x$. The associated locally constant system, $U$, on $\mathbf{B} \mathbb{Z}_{2}$ is the direct sum of the canonical line bundle and the trivial line bundle. Hence $A^{0} \otimes_{\mathbf{k}} U$, which corresponds to the space of cross sections, cannot be a free $A^{0}$ module. But $A^{0} \otimes_{\mathbf{k}} U$ is the fibre of the $A$ minimal model for the space $\mathbb{R} P^{3}$, viewed as a fibration $S^{3} \rightarrow \mathbb{R} P^{3} \rightarrow K\left(\mathbb{Z}_{2}, 1\right)$.

Proof of Proposition 7.1. By construction, $E_{\sigma}=\left(A(n) \otimes_{\sigma} \Lambda W\right) \otimes_{\mathbf{k}} \Lambda Z$. This identifies $E=A \otimes_{R} \Lambda Y$, with $\left(\Lambda Y, D_{0}\right)=A^{0} \otimes_{\mathbf{k}}(\Lambda Z, d)$. In particular (forgetting differentials), $E=A \otimes_{\mathbf{k}} \Lambda Z$ and hence is extendable.

It remains to prove that $(E, D)$ is an $A$-algebra; i.e. that $H(E, D)$ is a locally constant system. It is obviously sufficient to prove that the inclusion of a vertex $v$ in $\boldsymbol{\Delta}^{n}$ induces a quasi-isomorphism $A(n) \otimes_{\sigma}(\Lambda W \otimes \Lambda Z) \rightarrow \mathbf{k} \otimes_{v}(\Lambda W \otimes \Lambda Z)$. Rewrite this as a morphism $\varepsilon \otimes$ id $:\left(A(n) \otimes_{\mathbf{k}} \Lambda Z, \bar{\delta}\right) \rightarrow(\Lambda Z, d)$, where $\varepsilon: A(n) \rightarrow \mathbf{k}$ is the augmentation defined by $v$. In the generalized nilpotent case $\varepsilon \otimes$ id is a quasi-isomorphism because $\varepsilon$ is [21, Theorem 4.5].

Consider the case $\mathbf{k}=\mathbb{R}$ and $A(*)=A_{D R}(*)$. Let $\left(z_{i}\right)_{1 \leq i \leq N}$ be a basis of $Z^{p}$. The differential $\bar{\delta}$ has the form $\bar{\delta} z_{i}=\sum_{j=1}^{N} \theta_{i j} z_{j}+w\left(z_{i}\right)$, where $w\left(z_{i}\right) \in A_{D R}\left(\Delta^{n}\right) \otimes$ $\Lambda Z^{<p}$ and $\theta_{i j} \in A_{D R}^{1}\left(\Delta^{n}\right)$. The equation $\bar{\delta}^{2}=0$ implies that $\bar{\delta} \theta_{i j}=\sum_{k} \theta_{i k} \theta_{k j}$. Identify $z=\sum \lambda_{i} z_{i}$ with $\hat{z}=\left(\lambda_{1}, \ldots, \lambda_{N}\right)$. We are looking for an invertible matrix $M=\left(f_{i j}\right)_{1 \leq i, j \leq N}, f_{i j} \in A_{D R}^{0}\left(\Delta^{n}\right)$, such that $\bar{\delta}(M \hat{z}) \in A_{D R}\left(\Delta^{n}\right) \otimes \Lambda Z^{<p}$. This is provided by the solution of the differential equation, $\bar{\delta} M+M \theta=0, \theta=\left(\theta_{i j}\right)$, whose integrability condition is exactly $\bar{\delta} \theta=\theta \circ \theta$.

The last assertion of the proposition is an immediate consequence of the definitions. 
7.2. Formality. The notion of a formal space, whose rational homotopy type depends only on its cohomology algebra, has played a significant role in simply connected rational homotopy theory. This is in part because every simply connected space is a "perturbation" of the corresponding formal space.

We can now extend this notion to the non simply connected case. Indeed, let $X \in \mathcal{T}^{f}$ and consider the locally constant system $\mathcal{H}$ over $\mathbf{B} \boldsymbol{\pi}_{1}(X)$ corresponding to the $\pi_{1}(X)$-module, $H^{*}(\tilde{X} ; \mathbb{Q})$. Evidently $A_{P L} \otimes_{\mathbb{Q}}(\mathcal{H}, 0)$ is a 1 -connected $A_{P L^{-}}$ algebra of finite type; let $\left(A_{P L} \otimes_{R} \Lambda Y, D\right) \stackrel{\simeq}{\longrightarrow} A_{P L} \otimes_{\mathbb{Q}}(\mathcal{H}, 0)$ be its minimal model (Section 3). Thus the space $\left\langle A_{P L} \otimes_{R} \Lambda Y, D\right\rangle$ is a formal consequence of the group $\pi_{1}(X)$, the algebra $H^{*}(\tilde{X} ; \mathbb{Q})$ and the representation of $\pi_{1}(X)$ in $H^{*}(\tilde{X} ; \mathbb{Q})$. (Note that this construction could be applied to any representation of a discrete group $\Gamma$ in a suitable algebra, $H$.)

Definition 7.5. The space $\left\langle\left(A_{P L} \otimes_{R} \Lambda Y, D\right)\right\rangle$ is the formal space associated with $X$, and $X$ is formal if $\left\langle\left(A_{P L} \otimes_{R} \Lambda Y, D\right)\right\rangle \simeq X_{\mathbb{Q}}$ or equivalently, if $\left(A_{P L} \otimes_{R} \Lambda Y, D\right)$ is a minimal model for $X$.

If we consider instead the $\pi_{1}(X)$-module $C^{*}\left(\pi_{*}(\Omega \tilde{X}) \otimes \mathbb{Q}\right)$ where $\pi_{*}(\Omega \tilde{X}) \otimes \mathbb{Q}$ is the homotopy Lie algebra and $C^{*}(-)$ is the classical cochain construction of E. Cartan and D. Quillen, we get the corresponding notion of coformal space. In this context, we pose two problems:

Problem 1. Is every space the perturbation of a formal space as described in [14] in the simply connected case? If so, is there a well defined sequence of "obstructions to formality"? If so, are they independent of the ground field? Is there an analogue of grading automorphisms [21] Page 319]?

Problem 2. Are compact Kähler manifolds $M$ formal in the sense of Definition 7.3.1?

7.3. Universal fibrations. Let $F$ be a simply connected space in $\mathcal{T}^{f, 0}$. There is a universal fibration with fibre $F$, whose base is denoted $B A u t F$. Now on the one hand, a model of the universal cover of $B A u t F$ can be explicitly described (21, 22], [19]). On the other hand, $\pi_{1}(B A u t F)$ is just the group of homotopy classes of automorphisms of the Sullivan minimal model of $F$.

Problem 3. Find an explicit description of the $A_{P L}$-minimal model of BAutF.

7.4. Classification. One ultimate goal of an algebraisation of homotopy theory is to obtain an explicit determination of moduli spaces:

Problem 4. For given discrete group $\pi$ and given minimal Sullivan algebra $(\Lambda Z, d)$ classify explicitly:

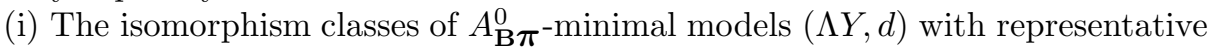
Sullivan algebra $(\Lambda Z, d)$;

(ii) For each $(\Lambda Y, d)$ the isomorphism classes of $A_{P L}$-minimal models $\left(A_{P L} \otimes_{R}\right.$ $\Lambda Y, D)$.

7.5. Nilpotent fibres. At various places in this paper we have relied heavily on the hypothesis that our fibres are simply connected. Since classical Sullivan theory works for nilpotent spaces we ask

Problem 5. Does the theory presented here extend to the case of nilpotent fibres? Note that a solution would give an interesting way of approaching solvmanifolds, since these fibre over a torus with fibre a nilmanifold [17, Theorem 2, Page 25]. 
7.6. Infinite type. The restriction that the rational cohomology of the universal cover have finite type is really unfortunate, since it rules out even simple spaces such as $S^{1} \vee S^{3}$. This is also a difficulty in the simply connected case where Sullivan [21, Page 303] has suggested the use of continuous duals as a solution.

Problem 6. Extend the theory (or find a replacement) to include all CW spaces.

7.7. Rational homotopy invariants. Since the minimal model $\left(A_{P L} \otimes_{R} \Lambda Y, D\right)$ of a space $X \in \mathcal{T}^{f}$ determines $X_{\mathbb{Q}}$ up to homotopy equivalence, it is natural to try to determine how to read off rational homotopy invariants of $X$ directly from the model. In the case of Lusternik-Schnirelmann category the solution 9 to this problem in the simply connected case has had a number of useful consequences. Thus

Problem 7. Given $X \in \mathcal{T}^{f}$ determine the LS category of $X_{\mathbb{Q}}$ in terms of the $A_{P L}$-minimal model.

\section{REFERENCES}

[1] A.K. Bousfield, V.K.A.M. Gugenheim, On PL DeRham theory and rational homotopy type, Memoirs of the Amer. Math. Soc. 179, (1976). MR 54:13906

[2] A.K. Bousfield, D.M. Kan, Homotopy Limits, Completions and Localisations, Lecture Notes 304, (1972), Springer Verlag. MR 51:1825

[3] E.H. Brown, R.H. Szczarba, Continuous cohomology and real homotopy type, Trans. Amer. Math. Soc. 311, (1989), 57-106. MR 89f:55005

[4] E.H. Brown, R.H. Szczarba, Rational and real homotopy theory with arbitrary fundamental groups, Duke Math. J. 71, (1993), 299-316. MR 94i:55017

[5] E.H. Brown, R.H. Szczarba, Continuous cohomology and real homotopy type II, Astérisque 191, (1990), 45-70. MR 92d:55004

[6] P. Deligne, P. Griffiths, J. Morgan, D. Sullivan, Real homotopy theory of Kähler manifolds, Inventiones Math. 29, (1975), 245-274. MR 52:3584

[7] A. Dold, Partitions of unity in the theory of fibrations, Ann. of Math. 78, (1963), 223-255. MR 27:5264

[8] S. Eilenberg, S. MacLane, Relations between homology and homotopy groups of spaces, Ann. of Math. 46, (1945), 480-509. MR 7:137g

[9] Y. Félix, S. Halperin, Rational LS-category and its applications, Trans. Amer. Math. Soc. 273, (1982), 1-37. MR 84h:55011

[10] Y. Félix, S. Halperin, J.-C. Thomas, Rational Homotopy and its Applications, in preparation.

[11] A. Gómez-Tato, Théorie de Sullivan pour la cohomologie à coefficients locaux, Trans. Amer. Math. Soc. 330, (1992), 235-305. MR 92f:55012

[12] J. O. Goyo, The Sullivan model of the homotopy-fixed-point set, Thesis, University of Toronto, (1989).

[13] S. Halperin, Lectures on minimal models, Mémoires S.M.F. Nouvelle série 9-10, (1983). MR 85i:55009

[14] S. Halperin, J. Stasheff, Obstructions to homotopy equivalences, Advances in Math. 32, (1979), 233-279. MR 80j:55016

[15] S. Halperin, D. Tanré, Fibrés $C^{\infty}$ et Homotopie filtrée, Illinois J. of Math., 34, (1990), 284-324. MR 91g:55018

[16] J.P. May, Simplicial objects in algebraic topology, Midway reprint, University Chicago Press, Chicago 1982. MR 93m:55025

[17] G.D. Mostow, Factor spaces of solvable groups, Ann. of Math. 60, (1954), 1-27. MR 15:853g. MR 19:752d

[18] D. Quillen, Rational homotopy theory, Ann. of Math. 90, (1969), 205-295. MR 41:2678

[19] S. Schlessinger, J. Stasheff, Deformation theory and rational homotopy type, Preprint.

[20] D. Sullivan, Geometric Topology, part I; Localization, Periodicity, and Galois Symmetry, M.I.T. Press, Cambridge, (1970). MR 58:13006a 
[21] D. Sullivan, Infinitesimal Computations in Topology, Publications I.H.E.S. 47, (1977), 269331. MR 58:31119

[22] D. Tanré, Homotopie rationnelle: Modèles de Chen, Quillen, Sullivan, Lecture Notes in Math. 1025, Springer-Verlag, (1983). MR 86b:55010

Departamento de Xeometría e Topoloxía, Universidade de Santiago de Compostela, Santiago de Compostela, 15706 España

E-mail address: agtato@zmat.usc.es

College of Computer, Mathematical and Physical Science, University of Maryland, College Park, Maryland 20742-3281

E-mail address: shalper@deans.umd.edu

U.R.A. CNRS 0751, U.F.R. De Mathématiques, Université des Sciences et Technologies de Lille, 59655 Villeneuve d'AscQ Cedex, France

E-mail address: Daniel.Tanre@agat.univ-lille1.fr 